Honam Mathematical J. 36 (2014), No. 2, pp. 305-338

http://dx.doi.org/10.5831/HMJ.2014.36.2.305

\title{
ARITHMETIC SUMS SUBJECT TO LINEAR AND CONGRUENT CONDITIONS AND SOME APPLICATIONS
}

\author{
Aeran Kim, Daeyeoul Kim* and \\ AYYADURAI SANKARANARAYANAN
}

\begin{abstract}
We investigate the explicit evaluation for the sum
$\sum_{(a, b, x, y) \in \mathbb{N}^{4},} a b$ in terms of various divisor functions (where $C(x, y)$ $a x+b y=n$,
$C(x, y)$

is the set of residue conditions on $x$ and $y$ ) for various fixed $C(x, y)$. We also obtain some identities and congruences as interesting applications.
\end{abstract}

\section{Introduction}

For $d, n, u \in \mathbb{N}$ and $s \in \mathbb{N} \cup\{0\}$, we define some divisor functions for our use in the sequel.

$$
\sigma_{s}(n)=\sum_{d \mid n} d^{s}, \quad \sigma_{s}^{*}(n ; u):=\sum_{\substack{d \mid n, \frac{n}{d} \not \equiv 0}} d^{s}, \quad \sigma_{s}^{*}(n):=\sigma_{s}^{*}(n ; 2)
$$

and we define the arithmetic function $c_{8}(n)$ by the expression:

$$
\sum_{n=1}^{\infty} c_{8}(n) q^{n}:=q \prod_{m=1}^{\infty}\left(1-q^{2 m}\right)^{4}\left(1-q^{4 m}\right)^{4} \text { with } q \in \mathbb{C},|q|<1 .
$$

For $k, n \in \mathbb{N}$, we define the arithmetic function $F_{k}(n)$ by

$$
F_{k}(n)= \begin{cases}1, & \text { if } k \mid n \\ 0, & \text { if } k \nmid n\end{cases}
$$

Received February 15, 2014. Accepted March 31, 2014.

2010 Mathematics Subject Classification. 33E20, 11A25, 11A41.

Key words and phrases. Liouville's identity, divisor functions, congruences.

${ }^{*}$ Corresponding author 
For $k \in \mathbb{N}$ and $n \in \mathbb{N}_{0}:=\mathbb{N} \cup\{0\}$, we denote the number of representations of $n$ as the sum of $k$ squares by $r_{k}(n)$, that is,

$$
r_{k}(n):=\operatorname{card}\left\{\left(x_{1}, \ldots, x_{k}\right) \in \mathbb{Z}^{k} \mid n=x_{1}^{2}+\cdots+x_{k}^{2}\right\} .
$$

As there is only one way of expressing 0 as a sum of $k$ squares, namely, $0=0^{2}+\cdots+0^{2}$, we have $r_{k}(0)=1$ for all $k \in \mathbb{N}$.

Let $n \in \mathbb{N}$ and let $f: \mathbb{Z} \rightarrow \mathbb{C}$ be an even function. In 1858 (see [21, p. 247], [22, p. 275]), Liouville asserted the surprising result that the sum

$$
S_{1}:=\sum_{\substack{(a, b, x, y) \in \mathbb{N}^{4}, a x+b y=n}}\{f(a-b)-f(a+b)\}
$$

can be expressed in terms of sums over the positive divisors $d$ of $n$. More precisely, we have

$$
S_{1}=f(0)\left(\sigma_{1}(n)-\sigma_{0}(n)\right)+\sum_{\substack{d \in \mathbb{N}, d \mid n}}\left(1+\frac{2 n}{d}-d\right) f(d)-2 \sum_{\substack{d \in \mathbb{N}, d \mid n}}\left(\sum_{v=1}^{d} f(v)\right) .
$$

In the literature, several proofs of (2) are available (for example see [23], [25], [27, p. 93] and [28]). The identity (2) of Liouville has a lot of important applications. Bouniakowsky's theorem on the number of specific representations of a prime number $q \equiv 7(\bmod 16)$, Besge's formula for the twisted convolution sums (for $s=1,2$ )(see [31]),

$$
A_{s}(n):=\sum_{\substack{k \in \mathbb{N}, k<n / s}} \sigma_{1}(k) \sigma_{1}(n-s k)
$$

are some of them to be quoted as applications. We note that (see [31])

$$
A_{1}(n)=\frac{5}{12} \sigma_{3}(n)+\left(\frac{1}{12}-\frac{1}{2} n\right) \sigma_{1}(n)
$$

and

$$
A_{2}(n)=\frac{1}{12} \sigma_{3}(n)+\frac{1}{3} \sigma_{3}\left(\frac{n}{2}\right)+\left(\frac{1}{24}-\frac{1}{8} n\right) \sigma_{1}(n)+\left(\frac{1}{24}-\frac{1}{4} n\right) \sigma_{1}\left(\frac{n}{2}\right) .
$$

The extension of the Liouville's identity (2) to three variables is due to McAfee and Williams (see [24, Theorem 1.2, p. 35]) and the four variable extension is due to Alaca, Alaca, McAfee and Williams (see [1, Theorem 6.4, p. 20]). In 2000, Huard, Ou, Spearman and Williams obtained a far reaching generalization of the Liouville's identity (2) (which is stated as Proposition 2.1 in the sequel). 
For $a, b, n \in \mathbb{N}$, we define the convolution sum

$$
S_{a, b}(n):=\sum_{m=1}^{n-1} \sigma_{a}(m) \sigma_{b}(n-m) .
$$

It is plain that $S_{a, b}(n)=S_{b, a}(n)$ for all $a, b, n \in \mathbb{N}$. Ramanujan showed that $S_{a, b}(n)$ can be evaluated in terms of $\sigma_{a+b+1}(n), \sigma_{a+b-1}(n), \cdots, \sigma_{3}(n)$, $\sigma_{1}(n)$ for the nine pairs $(a, b) \in \mathbb{N}^{2}$ satisfying $a+b=2,4,6,8,12, a \leq b$, $a \equiv b \equiv 1(\bmod 2)$.

As a nice application to their general identity, Huard, Ou, Spearman and Williams established that all these nine Ramanujan's evaluations can be obtained by suitable choices of the function $f(a, b, x, y)$ in their general identity. It should be noted that in a certain sense, the nine evaluations of $S_{a, b}(n)$ with $a$ and $b$ odd are the only ones. This fact was proved by Levitt (see [20]) using the theory of modular forms. An interesting and a some what difficult question is to investigate a sum of the sort :

$$
S_{1}^{*}:=\sum_{\begin{array}{c}
(a, b, x, y) \in \mathbb{N}^{4}, \\
a x+b y=n, \\
x \equiv l_{1}+\left(\bmod k_{1}\right), \\
y \equiv l_{2}\left(\bmod k_{2}\right)
\end{array}}\{f(a-b)-f(a+b)\},
$$

where, as before, $f: \mathbb{Z} \rightarrow \mathbb{C}$ is an even function and $n \in \mathbb{N}$. Here $k_{1}$ and $k_{2}$ are integers $\geq 2$.

Let $C(x, y):=\left\{(x, y) \mid x \equiv l_{1}\left(\bmod k_{1}\right), y \equiv l_{2}\left(\bmod k_{2}\right)\right\}$.

The aim of this paper is to obtain explicit evaluation in terms of divisor functions of the sum :

$$
S\left(l_{1}, l_{2} ; k_{1}, k_{2}\right):=\sum_{\begin{array}{c}
(a, b, x, y) \in \mathbb{N}^{4}, \\
a x+b y=n, \\
C(x, y)
\end{array}} a b
$$

for various fixed residue classes set $C(x, y)$. More precisely, we prove :

Theorem 1.1. For $n \in \mathbb{N}$, we have

\begin{tabular}{|c|c|c|}
\hline & $C(x, y)=$ & $\begin{array}{c}\sum_{(a, b, x, y) \in \mathbb{N}^{4}}, a b= \\
a x+b y=n, \\
C(x, y)\end{array}$ \\
\hline (i) & $x \equiv 0(\bmod 2), y \equiv 0(\bmod 2)$ & $\frac{1}{12}\left(5 \sigma_{3}\left(\frac{n}{2}\right)+(1-3 n) \sigma_{1}\left(\frac{n}{2}\right)\right)$ \\
\hline (ii) & $\begin{array}{c}x \not \equiv 0(\bmod 2), y \equiv 0(\bmod 2) \\
\text { or } x \equiv 0(\bmod 2), y \not \equiv 0(\bmod 2)\end{array}$ & $\frac{1}{24}\left(2 \sigma_{3}^{*}(n)+\sigma_{1}^{*}(n)-3 n \sigma_{1}(n)\right)$ \\
\hline (iii) & $x \not \equiv 0(\bmod 2), y \not \equiv 0(\bmod 2)$ & $\frac{1}{4}\left(\sigma_{3}^{*}(n)-n \sigma_{1}^{*}(n)\right)$ \\
\hline
\end{tabular}

TABLE 1. Formula for modulo 2 
Theorem 1.2. For $n \in \mathbb{N}$, we have

\begin{tabular}{|c|c|c|}
\hline & $C(x, y)=$ & $\sum_{\substack{(a, b, x, y) \in \mathbb{N}^{4}, a x+b y=n, C(x, y)}} a b=$ \\
\hline (i) & $x \equiv 0(\bmod 4), y \equiv 0(\bmod 4)$ & $\frac{1}{12}\left(5 \sigma_{3}\left(\frac{n}{4}\right)+\left(1-\frac{3}{2} n\right) \sigma_{1}\left(\frac{n}{4}\right)\right)$ \\
\hline (ii) & $x \equiv 2(\bmod 4), y \equiv 2(\bmod 4)$ & $\frac{1}{8}\left(2 \sigma_{3}^{*}\left(\frac{n}{2}\right)-n \sigma_{1}^{*}\left(\frac{n}{2}\right)\right)$ \\
\hline (iii) & $x \equiv 0(\bmod 4), y \equiv 2(\bmod 4)$ & $\frac{1}{24}\left\{2 \sigma_{3}^{*}\left(\frac{n}{2}\right)+\sigma_{1}^{*}\left(\frac{n}{2}\right)-\frac{3}{2} n \sigma_{1}\left(\frac{n}{2}\right)\right\}$ \\
\hline (iv) & $x \not \equiv 0(\bmod 4), y \neq \equiv 0(\bmod 4)$ & $\frac{1}{8}\left[3 \sigma_{3}^{*}(n)+2 \sigma_{3}^{*}\left(\frac{n}{2}\right)-3 n\left\{\sigma_{1}^{*}(n)+\sigma_{1}^{*}\left(\frac{n}{2}\right)\right\}\right]$ \\
\hline (v) & $x \equiv 0(\bmod 4), y \neq 0(\bmod 4)$ & $\frac{1}{48}\left[\sigma_{3}^{*}(n)+4 \sigma_{3}^{*}\left(\frac{n}{2}\right)\right.$ \\
& & $\left.-(3 n-2)\left\{\sigma_{1}^{*}(n)+\sigma_{1}^{*}\left(\frac{n}{2}\right)\right\}-9 n \sigma_{1}\left(\frac{n}{4}\right)\right]$ \\
\hline (vi) & $x \equiv 0(\bmod 4), y \equiv 1(\bmod 2)$ & $\frac{1}{48}\left[\sigma_{3}^{*}(n)-(3 n-2) \sigma_{1}^{*}(n)-6 n \sigma_{1}\left(\frac{n}{4}\right)\right]$ \\
\hline
\end{tabular}

TABLE 2. Formula for modulo 4 and 2

Theorem 1.3. For $n \in \mathbb{N}$, we have

(i)

$$
\sum_{\substack{a, b, x, y) \in \mathbb{N}^{4}, a x+b y=n \\ x \equiv 0 \quad(\bmod 2), y \equiv 2 \quad(\bmod 4)}} a b=\frac{1}{48}\left(16 \sigma_{3}^{*}\left(\frac{n}{2}\right)-2(3 n-1) \sigma_{1}^{*}\left(\frac{n}{2}\right)-3 n \sigma_{1}\left(\frac{n}{2}\right)\right) .
$$

(ii)

$$
\sum_{\substack{(a, b, x, y) \in \mathbb{N}^{4}, a x+b y=n, x \equiv 0 \quad(\bmod 4), y \equiv 0 \quad(\bmod 2)}} a b=\frac{1}{24}\left(2 \sigma_{3}\left(\frac{n}{2}\right)+8 \sigma_{3}\left(\frac{n}{4}\right)+\frac{1}{2}(2-3 n) \sigma_{1}\left(\frac{n}{2}\right)+(1-3 n) \sigma_{1}\left(\frac{n}{4}\right)\right) .
$$

Theorem 1.4. For $n \in \mathbb{N}$, we have

\begin{tabular}{|c|c|c|}
\hline & $C(x, y)=$ & $\sum_{\substack{(a, b, x, y) \in \mathbb{N}^{4}, a x+b y=n \\
a(b, y)}} a b=$ \\
\hline (i) & $x \equiv 0(\bmod 8), y \equiv 0(\bmod 4)$ & $\frac{1}{96}\left[8\left\{\sigma_{3}\left(\frac{n}{4}\right)+4 \sigma_{3}\left(\frac{n}{8}\right)\right\}-(3 n-4) \sigma_{1}\left(\frac{n}{4}\right)\right.$ \\
& & $\left.-2(3 n-2) \sigma_{1}\left(\frac{n}{8}\right)\right]$ \\
\hline (ii) & $x \equiv 4(\bmod 8), y \equiv 0(\bmod 4)$ & $\frac{1}{96}\left[32 \sigma_{3}^{*}\left(\frac{n}{4}\right)-(9 n-4) \sigma_{1}^{*}\left(\frac{n}{4}\right)-3 n \sigma_{1}\left(\frac{n}{8}\right)\right]$ \\
\hline (iii) & $x \equiv 0(\bmod 8), y \equiv 0(\bmod 2)$ & $\frac{1}{48}\left(\sigma_{3}\left(\frac{n}{2}\right)+\left(2-\frac{3 n}{2}\right) \sigma_{1}\left(\frac{n}{2}\right)+3 \sigma_{3}\left(\frac{n}{4}\right)\right.$ \\
& & $\left.+16 \sigma_{3}\left(\frac{n}{8}\right)+(2-6 n) \sigma_{1}\left(\frac{n}{8}\right)\right)$ \\
\hline (iv) & $x \equiv 0(\bmod 8), y \equiv 2(\bmod 4)$ & $\frac{1}{96}\left\{2 \sigma_{3}^{*}\left(\frac{n}{2}\right)-(3 n-4) \sigma_{1}^{*}\left(\frac{n}{2}\right)-6 n \sigma_{1}\left(\frac{n}{8}\right)\right\}$ \\
\hline (v) & $x \equiv 4(\bmod 8), y \equiv 0(\bmod 2)$ & $\frac{1}{96}\left\{6 \sigma_{3}^{*}\left(\frac{n}{2}\right)+32 \sigma_{3}^{*}\left(\frac{n}{4}\right)-3 n \sigma_{1}\left(\frac{n}{2}\right)\right.$ \\
& & $\left.-4(3 n-1) \sigma_{1}^{*}\left(\frac{n}{4}\right)\right\}$ \\
\hline (vi) & $x \equiv 4(\bmod 8), y \equiv 2(\bmod 4)$ & $\frac{1}{32}\left\{2 \sigma_{3}^{*}\left(\frac{n}{2}\right)-n \sigma_{1}^{*}\left(\frac{n}{2}\right)-2 n \sigma_{1}^{*}\left(\frac{n}{4}\right)\right\}$ \\
\hline
\end{tabular}

TABLE 3. Formula for modulo 8, 4 and 2 
Theorem 1.5. For $n \in \mathbb{N}$, we have

\begin{tabular}{|c|c|c|}
\hline & $C(x, y)=$ & $\sum_{\substack{(a, b, x, y) \in \mathbb{N}^{4}, a x+b y=n, C(x, y)}} a b=$ \\
\hline (i) & $x \equiv 0(\bmod 8), y \equiv 0(\bmod 8)$ & $\frac{1}{48}\left\{20 \sigma_{3}\left(\frac{n}{8}\right)-(3 n-4) \sigma_{1}\left(\frac{n}{8}\right)\right\}$ \\
\hline (ii) & $x \equiv 4(\bmod 8), y \equiv 0(\bmod 8)$ & $\frac{1}{96}\left\{8 \sigma_{3}^{*}\left(\frac{n}{4}\right)+4 \sigma_{1}^{*}\left(\frac{n}{4}\right)-3 n \sigma_{1}\left(\frac{n}{4}\right)\right\}$ \\
\hline (iii) & $x \equiv 4(\bmod 8), y \equiv 4(\bmod 8)$ & $\frac{1}{16}\left\{4 \sigma_{3}^{*}\left(\frac{n}{4}\right)-n \sigma_{1}^{*}\left(\frac{n}{4}\right)\right\}$ \\
\hline (iv) & $x \not \equiv 0(\bmod 8), y \not \equiv 0(\bmod 8)$ & $\frac{1}{32}\left[13 \sigma_{3}^{*}(n)+12 \sigma_{3}^{*}\left(\frac{n}{2}\right)+8 \sigma_{3}^{*}\left(\frac{n}{4}\right)\right.$ \\
& & $\left.-14 n\left\{\sigma_{1}^{*}(n)+\sigma_{1}^{*}\left(\frac{n}{2}\right)+\sigma_{1}^{*}\left(\frac{n}{4}\right)\right\}+c_{8}(n)\right]$ \\
\hline (v) & $x \equiv 0(\bmod 8), y \not \equiv 0(\bmod 8)$ & $\frac{1}{192}\left\{\sigma_{3}^{*}(n)+4 \sigma_{3}^{*}\left(\frac{n}{2}\right)\right.$ \\
& & $+16 \sigma_{3}^{*}\left(\frac{n}{4}\right)-2(3 n-4) \sigma_{1}(n)$ \\
& & $\left.-4(9 n+2) \sigma_{1}\left(\frac{n}{8}\right)-3 c_{8}(n)\right\}$ \\
\hline
\end{tabular}

\section{TABLE 4. Formula for modulo 8}

Theorem 1.6. For $n \in \mathbb{N}$, we have

$$
\sum_{\substack{(a, b, x, y) \in \mathbb{N}^{4}, a x+b y=n, x \equiv 0 \quad(\bmod 8), y \equiv 1 \quad(\bmod 2)}} a b=\frac{1}{192}\left\{\sigma_{3}^{*}(n)-2(3 n-4) \sigma_{1}^{*}(n)-24 n \sigma_{1}\left(\frac{n}{8}\right)-3 c_{8}(n)\right\} .
$$

As applications to the above theorems, we obtain explicit formulae for several twisted convolution sums (see Theorem 4.8 in section 4); we establish that the congruence relation

$$
\sum_{k=1}^{2 n-1} c_{8}(k) c_{8}(2 n-k) \equiv \sigma_{3}^{*}(n) \quad(\bmod 8)
$$

and the identity

$$
\sum_{k=1}^{2 n} c_{8}(k) c_{8}(2 n+1-k)=0
$$

hold. We also prove several congruence relations in connection with the arithmetic function $c(n)$ where $c(n)$ is given by the expression :

$$
\sum_{n=1}^{\infty} c(n) q^{n}:=q \prod_{m=1}^{\infty}\left(1-q^{m}\right)^{8}\left(1-q^{2 m}\right)^{8} \text { with } q \in \mathbb{C},|q|<1 .
$$

As an another interesting application, we obtain the identity :

$$
r_{8}(4 n)=16\left\{56 \sigma_{3}^{*}(n)+15 \sigma_{3}(n)\right\}
$$

which holds for all $n \in \mathbb{N}$.

For results closely related to the content of the paper, we refer to Glaisher [10, 11, 12]; B. C. Berndt [4]; H. Hahn [13]; J. G. Huard, Z. M. Ou, B. K. Spearman, and K. S. Williams [14]; G. Melfi [26]; B. Cho, D. 
Kim, and J.-K. Koo [6, 7, 8]; D. Kim, A. Kim, and N. Y. Ikikardes [18]; D. Kim, M.-S. Kim [19]; and A. Alaca, S. Alaca, and K. S. Williams $[2,3]$.

\section{A more general Liouville sum}

In 2000 Huard, Ou, Spearman and Williams proved a far reaching generalization of Liouville's identity. We state this generalization below as :

Proposition 2.1. Let $f: \mathbb{Z}^{4} \rightarrow \mathbb{C}$ be such that

(3) $f(a, b, x, y)-f(x, y, a, b)=f(-a,-b, x, y)-f(x, y,-a,-b)$

for all integers $a, b, x$ and $y$. For $n \in \mathbb{N}$, we have

$$
\begin{aligned}
& \sum_{\substack{(a, b, x, y) \in \mathbb{N}^{4}, a x+b y=n}}\{f(a, b, x,-y)-f(a,-b, x, y)+f(a, a-b, x+y, y) \\
& \quad-f(a, a+b, y-x, y)+f(b-a, b, x, x+y)-f(a+b, b, x, x-y)\} \\
& =\sum_{\substack{d \in \mathbb{N}, d \mid n}} \sum_{x \in \mathbb{N},}\left\{f\left(0, \frac{n}{d}, x, d\right)+f\left(\frac{n}{d}, 0, d, x\right)+f\left(\frac{n}{d}, \frac{n}{d}, d-x,-x\right)\right. \\
& \left.\quad-f\left(x, x-d, \frac{n}{d}, \frac{n}{d}\right)-f\left(x, d, 0, \frac{n}{d}\right)-f\left(d, x, \frac{n}{d}, 0\right)\right\} .
\end{aligned}
$$

Proof. See [31, p. 137].

\section{Proof of theorems}

\section{series Proof of the Theorem 1.1. :}

(i) We note that

$$
\sum_{\substack{(a, b, x, y) \in \mathbb{N}^{4}, a x+b y=n, x \equiv 0 \quad(\bmod 2), y \equiv 0 \quad(\bmod 2)}} a b=\sum_{\substack{\left(a, b, x^{\prime}, y^{\prime}\right) \in \mathbb{N}^{4}, 2 a x^{\prime}+2 b y^{\prime}=n}} a b=\sum_{\begin{array}{c}
\left(a, b, x^{\prime}, y^{\prime}\right) \in \mathbb{N}^{4}, \\
a x^{\prime}+b y^{\prime}=\frac{n}{2}
\end{array}} a b=A_{1}\left(\frac{n}{2}\right) .
$$

(ii) Let us take $f(a, b, x, y)=a b F_{2}(x)$ in Proposition 2.1. Then (3) is satisfied, so the left hand side becomes 


$$
\begin{aligned}
& \sum_{\substack{(a, b, x, y) \in \mathbb{N}^{4}, a x+b y=n}}\left\{a b F_{2}(x)+a b F_{2}(x)+a(a-b) F_{2}(x+y)\right. \\
& \left.-a(a+b) F_{2}(y-x)+(b-a) b F_{2}(x)-(a+b) b F_{2}(x)\right\} \\
& =\sum_{\substack{(a, b, x, y) \in \mathbb{N}^{4}, a x+b y=n}}\left\{-a b F_{2}(x+y)-a b F_{2}(y-x)\right\} \\
& =-2 \sum_{\substack{(a, b, x, y) \in \mathbb{N}^{4}, a x+b y=n, x \equiv 0 \quad(\bmod 2), y \equiv 0 \quad(\bmod 2)}} a b-2 \sum_{\begin{array}{c}
(a, b, x, y) \in \mathbb{N}^{4}, \\
a x+b y=n, \\
x \neq 0 \quad(\bmod 2), \\
y \neq 0 \quad(\bmod 2)
\end{array}} a b .
\end{aligned}
$$

We note that

$$
\begin{aligned}
& \sum_{\begin{array}{c}
(a, b, x, y) \in \mathbb{N}^{4}, \\
a x+b y=n, \\
x \neq 0 \quad(\bmod 2), \\
y \neq 0 \quad(\bmod 2)
\end{array}} a b=\sum_{\begin{array}{c}
(a, b, x, y) \in \mathbb{N}^{4}, \\
a x+b y=n
\end{array}} a b-\sum_{\begin{array}{c}
(a, b, x, y) \in \mathbb{N}^{4}, \\
a x+b y=n, \\
0 \equiv 0 \quad(\bmod 2), \\
y \neq 0 \quad(\bmod 2)
\end{array}} a b-\sum_{\begin{array}{c}
(a, b, x, y) \in \mathbb{N}^{4}, \\
a x+b y=n, \\
x \neq 0 \quad(\bmod 2), \\
y \equiv 0 \quad(\bmod 2)
\end{array}} a b \\
& \sum_{\substack{(a, b, x, y) \in \mathbb{N}^{4}, a x+b y=n, x \equiv 0 \quad(\bmod 2), y \equiv 0 \quad(\bmod 2)}} a b \\
& =\sum_{\begin{array}{c}
(a, b, x, y) \in \mathbb{N}^{4}, \\
a x+b y=n
\end{array}} a b-\sum_{\begin{array}{c}
(a, b, x, y) \in \mathbb{N}^{4}, \\
a x+b y=n, \\
x \equiv 0 \quad(\bmod 2)
\end{array}} a b-\sum_{\begin{array}{c}
(a, b, x, y) \in \mathbb{N}^{4}, \\
a x+b y=n, \\
x \neq 0 \quad(\bmod 2), \\
y \equiv 0 \quad(\bmod 2)
\end{array}} a b \\
& \begin{array}{c}
=A_{1}(n)-A_{2}(n)-\sum_{\substack{(a, b, x, y) \in \mathbb{N}^{4}, a x+b y=n, x \neq 0 \quad(\bmod 2), y \equiv 0 \quad(\bmod 2)}} a b .
\end{array}
\end{aligned}
$$

Therefore the right hand side of (4) is equal to

$$
2 \sum_{\substack{(a, b, x, y) \in \mathbb{N}^{4}, a x+b y=n, x \neq 0 \quad(\bmod 2), y \equiv 0 \quad(\bmod 2)}} a b-2 A_{1}\left(\frac{n}{2}\right)-2 A_{1}(n)+2 A_{2}(n) .
$$

However, the right hand side of Proposition 2.1 is 
$(6)$

$\sum_{\substack{d \in \mathbb{N}, d \mid n}} \sum_{\substack{x \in \mathbb{N}, x<d}}\left(\left(\frac{n}{d}\right)^{2} F_{2}(d-x)-x(x-d) F_{2}\left(\frac{n}{d}\right)-x d F_{2}(0)-d x F_{2}\left(\frac{n}{d}\right)\right)$

$=\sum_{\substack{d \in \mathbb{N}, d \mid n}} \sum_{\substack{x \in \mathbb{N} \\ x<d}}\left(\left(\frac{n}{d}\right)^{2} F_{2}(d-x)-x^{2} F_{2}\left(\frac{n}{d}\right)-x d\right)$

since 0 is divisible by 2 and it applies that $F_{2}(0)=1$. Then the first sum in (6) is

$$
\begin{aligned}
& \sum_{\substack{d \in \mathbb{N}, d \mid n}} \sum_{\substack{x \in \mathbb{N} \\
x<d}}\left(\frac{n}{d}\right)^{2} F_{2}(d-x) \\
& =\sum_{\substack{d|n, 2| d}}\left(\frac{n}{d}\right)^{2} \sum_{x=1}^{d-1} F_{2}(d-x)+\sum_{\substack{d \mid n, 2 \nmid d}}\left(\frac{n}{d}\right)^{2} \sum_{x=1}^{d-1} F_{2}(d-x) \\
& =\sum_{\substack{d|n, 2| d}}\left(\frac{n}{d}\right)^{2} \sum_{\substack{x=1 \\
2 \mid x}}^{d-1} 1+\sum_{\substack{d \mid n \\
2 \nmid d}}\left(\frac{n}{d}\right)^{2} \sum_{\substack{x=1 \\
2 \nmid x}}^{d-1} 1 \\
& =\sum_{\substack{d|n, 2| d}}\left(\frac{n}{d}\right)^{2}\left(\frac{d}{2}-1\right)+\sum_{\substack{d \mid n, 2 \nmid d}}\left(\frac{n}{d}\right)^{2}\left(\frac{d-1}{2}\right) .
\end{aligned}
$$

Writing $e=\frac{n}{d}$, then the right hand side of (7) is

$$
\begin{aligned}
= & \sum_{\substack{\frac{n}{e}|n, 2| \frac{n}{e}}} e^{2}\left(\frac{1}{2} \cdot \frac{n}{e}-1\right)+\frac{1}{2} \sum_{\substack{\frac{n}{e} \mid n, 2 \nmid \frac{n}{e}}} e^{2}\left(\frac{n}{e}-1\right) \\
= & \frac{1}{2} \sum_{\substack{\frac{n}{e}|n, 2| \frac{n}{e}}} n e-\sum_{\substack{\frac{n}{e}|n, 2| \frac{n}{e}}} e^{2}+\frac{1}{2}\left\{\sum_{\frac{n}{e} \mid n}\left(n e-e^{2}\right)-\sum_{\substack{\frac{n}{e}|n, 2| \frac{n}{e}}}\left(n e-e^{2}\right)\right\} \\
= & \frac{1}{2}\left\{n \sum_{e \mid n} e-\sum_{e \mid n} e^{2}-\sum_{e \mid \frac{n}{2}} e^{2}\right\} \\
= & \frac{1}{2}\left\{n \sigma_{1}(n)-\sigma_{2}(n)-\sigma_{2}\left(\frac{n}{2}\right)\right\} .
\end{aligned}
$$


The second sum in (6) is

$$
\begin{aligned}
\sum_{\substack{d \in \mathbb{N} \\
d \mid n}} \sum_{\substack{x \in \mathbb{N}, x<d}} x^{2} F_{2}\left(\frac{n}{d}\right) & =\sum_{d \mid n} F_{2}\left(\frac{n}{d}\right) \sum_{x=1}^{d-1} x^{2} \\
& =\sum_{d \mid n} F_{2}\left(\frac{n}{d}\right) \cdot \frac{(d-1) d(2 d-1)}{6} \\
& =\frac{1}{6} \sum_{d \mid \frac{n}{2}}\left(2 d^{3}-3 d^{2}+d\right) \\
& =\frac{1}{3} \sigma_{3}\left(\frac{n}{2}\right)-\frac{1}{2} \sigma_{2}\left(\frac{n}{2}\right)+\frac{1}{6} \sigma_{1}\left(\frac{n}{2}\right) .
\end{aligned}
$$

Finally, the third sum in (6) is

$$
\begin{aligned}
\sum_{\substack{d \in \mathbb{N}, d \mid n}} \sum_{\substack{x \in \mathbb{N}, x<d}} x d & =\sum_{d \mid n} d \sum_{x=1}^{d-1} x=\sum_{d \mid n} d \cdot \frac{(d-1) d}{2}=\frac{1}{2} \sum_{d \mid n}\left(d^{3}-d^{2}\right) \\
& =\frac{1}{2}\left\{\sigma_{3}(n)-\sigma_{2}(n)\right\} .
\end{aligned}
$$

Therefore the right hand side of (6) is

$$
\frac{n}{2} \sigma_{1}(n)-\frac{1}{3} \sigma_{3}\left(\frac{n}{2}\right)-\frac{1}{6} \sigma_{1}\left(\frac{n}{2}\right)-\frac{1}{2} \sigma_{3}(n) .
$$

Equating (5) with (8) we obtain the formula $\sum_{(a, b, x, y) \in \mathbb{N}^{4},} a b$

$$
\begin{gathered}
(a, b, x, y) \in \mathbb{N}^{4}, \\
a x+b y=n, \\
x \neq 0(\bmod 2), \\
y \equiv 0(\bmod 2)
\end{gathered}
$$

For the proof of $\quad \sum \quad a b$, we observe that

$$
\begin{gathered}
(a, b, x, y) \in \mathbb{N}^{4}, \\
a x+b y=n, \\
x \equiv 0(\bmod 2), \\
y \neq 0(\bmod 2)
\end{gathered}
$$

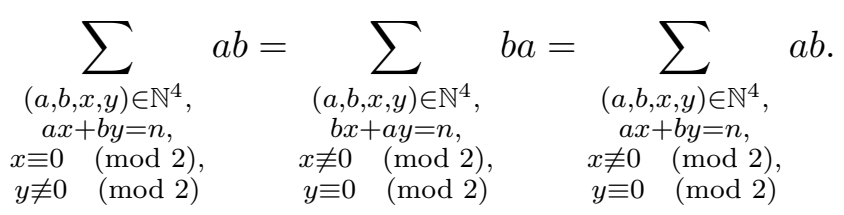


(iii) The sum $\sum_{(a, b, y) \in \mathbb{N}^{4}} a b$ can be separated as

$$
\begin{gathered}
(a, b, x, y) \in \mathbb{N}^{4}, \\
a x+b y=n, \\
x \neq 0(\bmod 2), \\
y \neq 0(\bmod 2)
\end{gathered}
$$

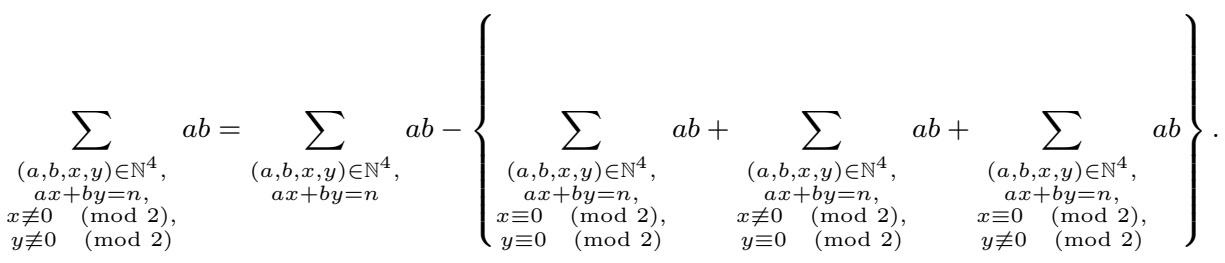

Thus we use (i), (ii) of Theorem 1.1.

Proof of the Theorem 1.2. :

(i) We can deduce that

$$
\sum_{\substack{(a, b, x, y) \in \mathbb{N}^{4}, a x+b y=n, x \equiv 0 \quad(\bmod 4), y \equiv 0 \quad(\bmod 4)}} a b=\sum_{\begin{array}{c}
\left(a, b, x^{\prime}, y^{\prime}\right) \in \mathbb{N}^{4}, \\
4 a x^{\prime}+4 b y^{\prime}=n
\end{array}} a b=\sum_{\begin{array}{c}
\left(a, b, x^{\prime}, y^{\prime}\right) \in \mathbb{N}^{4}, \\
a x^{\prime}+b y^{\prime}=\frac{n}{4}
\end{array}} a b=A_{1}\left(\frac{n}{4}\right) .
$$

(ii) We choose $f(a, b, x, y)=a b F_{2}(x) F_{4}(y)$ in Proposition 2.1, and note that the condition (3) is satisfied. So the left hand side of Proposition 2.1 becomes (since $F_{4}(y)=F_{4}(-y)$ ),

(9)

$$
\begin{aligned}
& \sum_{\substack{(a, b, x, y) \in \mathbb{N}^{4}, a x+b y=n}}\left(2 a b F_{2}(x) F_{4}(y)+a(a-b) F_{2}(x+y) F_{4}(y)-a(a+b) F_{2}(y-x) F_{4}(y)\right. \\
& \left.+(b-a) b F_{2}(x) F_{4}(x+y)-(a+b) b F_{2}(x) F_{4}(x-y)\right) \\
& =\sum_{\substack{(a, b, x, y) \in \mathbb{N}^{4}, a x+b y=n}}\left\{2 a b F_{4}(y)+a(a-b) F_{4}(y)-a(a+b) F_{4}(y)\right. \\
& a x+b y=n \text {, } \\
& x \equiv 0 \quad(\bmod 2) \\
& y \equiv 0 \quad(\bmod 2) \\
& \left.+(b-a) b F_{4}(x+y)-(a+b) b F_{4}(x-y)\right\} \\
& =-2 \sum_{\substack{(a, b, x, y) \in \mathbb{N}^{4}, a x+b y=n,}} a b-2 \sum_{\begin{array}{c}
(a, b, x, y) \in \mathbb{N}^{4}, \\
a x+b y=n,
\end{array}} \\
& x \equiv 0 \quad(\bmod 4), \quad x \equiv 2 \quad(\bmod 4), \\
& y \equiv 0 \quad(\bmod 4) \quad y \equiv 2 \quad(\bmod 4)
\end{aligned}
$$

When $x \equiv 1(\bmod 2)$ and $y \equiv 1(\bmod 2)$, note that we have used $F_{4}(y)=0$ and $F_{2}(x)=0$.

Then the left part of this proof is similar to the previous (ii) of Theorem 1.1. 
(iii) We observe that

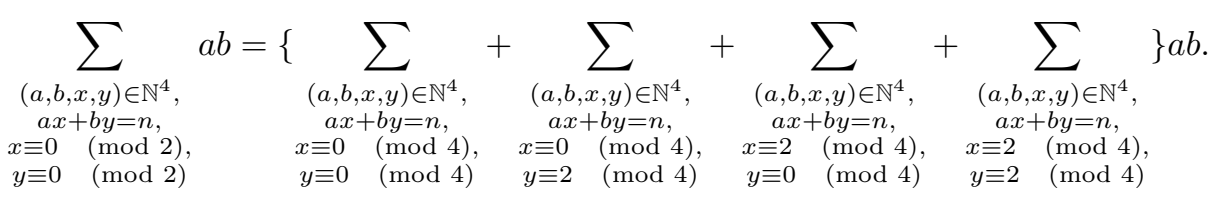

Thus

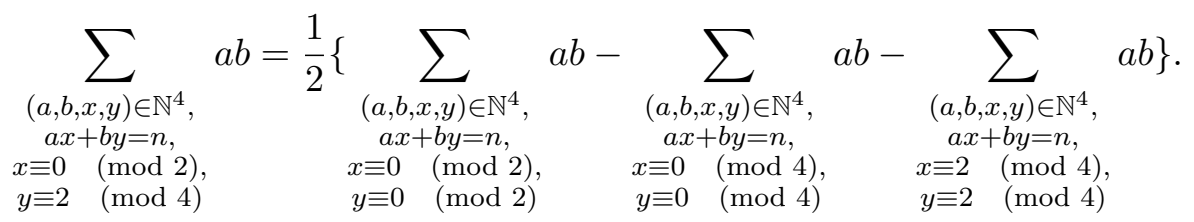

So we refer to (i) of Theorem 1.1, (i) and (ii) of Theorem 1.2.

(iv) We have

$\sum_{\substack{(a, b, x, y) \in \mathbb{N}^{4}, a x+b y=n, x \neq 0(\bmod 4), y \neq 0 \quad(\bmod 4)}} a b=\sum_{k=1}^{n-1}\left(\sum_{\substack{a \mid k, 4 \nmid \frac{k}{a}}} a\right)\left(\sum_{\substack{b \mid n-k, 4 \nmid\left(\frac{n-k}{b}\right)}} b\right)$

$=\sum_{k=1}^{n-1}\left(\sum_{\substack{a|k \\ 4| \frac{k}{a}}} a-\sum_{\substack{a|k, b| n-k}} b-\sum_{\substack{b|n-k, 4|\left(\frac{n-k}{b}\right)}} b\right)$

$=\sum_{k=1}^{n-1}\left(\sigma_{1}(k)-\sigma_{1}\left(\frac{k}{4}\right)\right)\left(\sigma_{1}(n-k)-\sigma_{1}\left(\frac{n-k}{4}\right)\right)$

$=\sum_{k=1}^{n-1} \sigma_{1}(k) \sigma_{1}(n-k)-2 \sum_{k<\frac{n}{4}} \sigma_{1}(k) \sigma_{1}(n-4 k)+\sum_{k<\frac{n}{4}} \sigma_{1}(k) \sigma_{1}\left(\frac{n}{4}-k\right)$

$=A_{1}(n)-2 A_{4}(n)+A_{1}\left(\frac{n}{4}\right)$

and we use

$$
\begin{aligned}
A_{4}(n)=\sum_{k<n / 4} \sigma(k) \sigma(n-4 k) \\
=\frac{1}{48}\left(\sigma_{3}(n)+(2-3 n) \sigma_{1}(n)+3 \sigma_{3}\left(\frac{n}{2}\right)+16 \sigma_{3}\left(\frac{n}{4}\right)\right. \\
\left.\quad \quad+(2-12 n) \sigma_{1}\left(\frac{n}{4}\right)\right)
\end{aligned}
$$


from [14, Theorem 4].

(v) Since

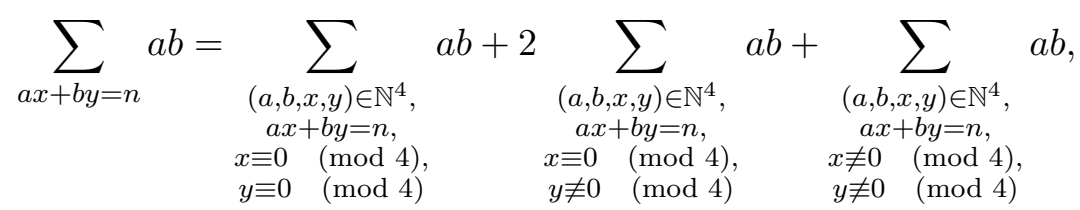

we obtain

$$
\sum_{\substack{a, b, x, y) \in \mathbb{N}^{4}, a x+b y=n, x \equiv 0 \quad(\bmod 4), y \neq 0 \quad(\bmod 4)}} a b=\frac{1}{2}\left\{A_{1}(n)-A_{1}\left(\frac{n}{4}\right)-\sum_{\begin{array}{r}
(a, b, x, y) \in \mathbb{N}^{4}, \\
a x+b y=n, \\
x \neq 0 \quad(\bmod 4), \\
y \neq 0 \quad(\bmod 4)
\end{array}} a b\right\} .
$$

Now, we use (i) and (iv) of Theorem 1.2 to obtain the result.

(vi) Let us consider

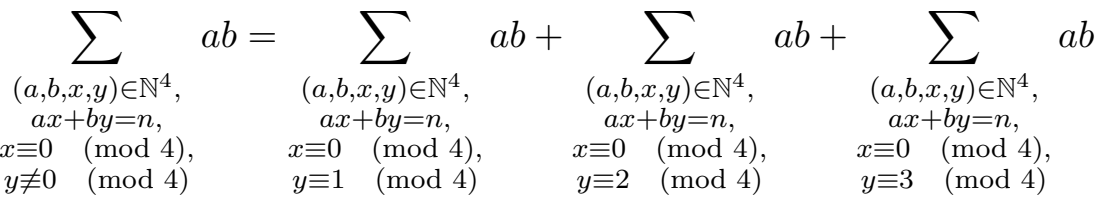

$$
\begin{aligned}
& =\sum_{\substack{(a, b, x, y) \in \mathbb{N}^{4}, a x+b y=n, x \equiv 0 \quad(\bmod 4),}} a b+\sum_{\substack{(a, b, x, y) \in \mathbb{N}^{4}, a x+b y=n, x \equiv 0 \quad(\bmod 4), y \equiv 1 \quad(\bmod 2)}}^{y \equiv 2 \quad(\bmod 4)}
\end{aligned}
$$

Note that both the conditions $y \equiv 1(\bmod 4)$ and $y \equiv 3(\bmod 4)$ are merged in $y \equiv 1(\bmod 2)$. Then we use (iii) and $(\mathrm{v})$ of Theorem 1.2 to obtain the result.

Proof of the Theorem 1.3. :

(i) We note that

$$
\sum_{\substack{(a, b, x, y) \in \mathbb{N}^{4}, a x+b y=n, x \equiv 0 \quad(\bmod 2),}} a b=\sum_{\substack{(a, b, x, y) \in \mathbb{N}^{4}, a x+b y=n, x \equiv 2(\bmod 4),}} a b+\sum_{\begin{array}{c}
(a, b, x, y) \in \mathbb{N}^{4}, \\
a x+b y=n, \\
y \equiv 0 \quad(\bmod 4,) \\
y \equiv 2(\bmod 4)
\end{array}} a b .
$$

Therefore (i) follows from (ii) and (iii) of Theorem 1.2.

(ii) Follows in a similar fashion using (i) and (iii) of Theorem 1.2. 


\section{Proof of the Theorem 1.4. :}

(i) It is easily checked that

$$
\begin{aligned}
& \sum_{\substack{(a, b, x, y) \in \mathbb{N}^{4}, a x+b y=n, x \equiv 0 \quad(\bmod 8), y \equiv 0 \quad(\bmod 4)}} a b=\sum_{\substack{(a, b, x, y) \in \mathbb{N}^{4}, 8 a x+4 b y=n}} a b=\sum_{\substack{(a, b, x, y) \in \mathbb{N}^{4}, 2 a x+b y=\frac{n}{4}}} a b \\
& =\sum_{k<\frac{n}{8}}\left(\sum_{a \mid k} a\right)\left(\sum_{b \mid\left(\frac{n}{4}-2 k\right)} b\right)=\sum_{k<\frac{n}{8}} \sigma_{1}(k) \sigma_{1}\left(\frac{n}{4}-2 k\right)=A_{2}\left(\frac{n}{4}\right) .
\end{aligned}
$$

(ii) Since

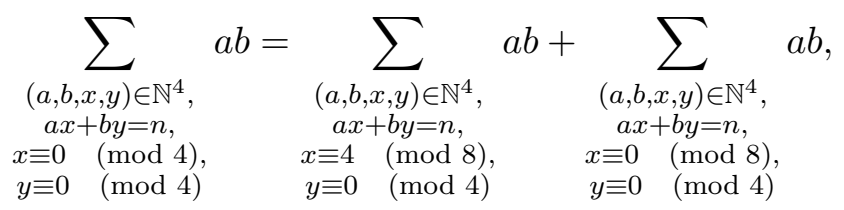

we use (i) of Theorem 1.4 and (i) of Theorem 1.2 to obtain the result.

(iii) We write

$$
\sum_{\substack{(a, b, x, y) \in \mathbb{N}^{4}, a x+b y=n, x \equiv 0 \quad(\bmod 8), y \equiv 0 \quad(\bmod 2)}} a b=\sum_{\begin{array}{c}
(a, b, x, y) \in \mathbb{N}^{4}, \\
8 a x+2 b y=n
\end{array}} a b=\sum_{\begin{array}{c}
(a, b, x, y) \in \mathbb{N}^{4}, \\
4 a x+b y=\frac{n}{2}
\end{array}} a b
$$

$$
=\sum_{k<\frac{n}{8}}\left(\sum_{a \mid k} a\right)\left(\sum_{b \mid\left(\frac{n}{2}-4 k\right)} b\right)=\sum_{k<\frac{n}{8}} \sigma_{1}(k) \sigma_{1}\left(\frac{n}{2}-4 k\right)=A_{4}\left(\frac{n}{2}\right),
$$

and so we use (10) to obtain the result.

(iv) Note that

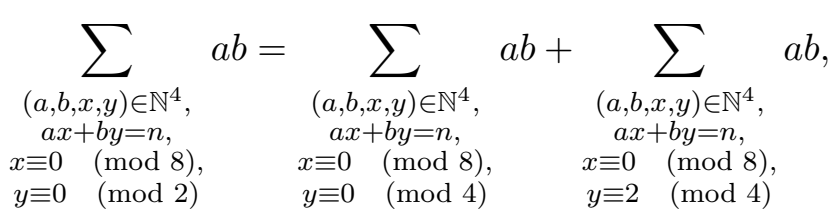

and hence, we use (i) and (iii) of Theorem 1.4 to obtain the result. 
(v) We observe that

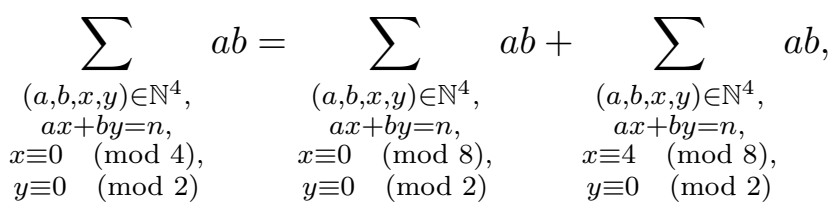

and so we use (ii) of Theorem 1.3 and (iii) of Theorem 1.4 to obtain the result.

(vi) Since

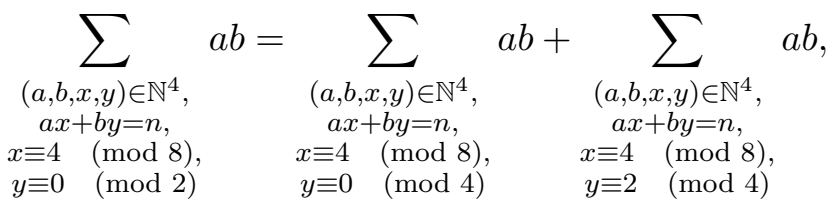

the result follows from (ii) and (v) of Theorem 1.4.

Proof of the Theorem 1.5. :

(i) Follows from

$$
\sum_{\substack{(a, b, x, y) \in \mathbb{N}^{4}, a x+b y=n, x \equiv 0 \quad(\bmod 8), y \equiv 0 \quad(\bmod 8)}} a b=\sum_{\substack{(a, b, x, y) \in \mathbb{N}^{4}, 8 a x+8 b y=n}} a b=\sum_{\begin{array}{c}
(a, b, x, y) \in \mathbb{N}^{4}, \\
a x+b y=\frac{n}{8}
\end{array}} a b=A_{1}\left(\frac{n}{8}\right) .
$$

(ii) We subtract (i) of Theorem 1.5 from (i) of Theorem 1.4. That is,

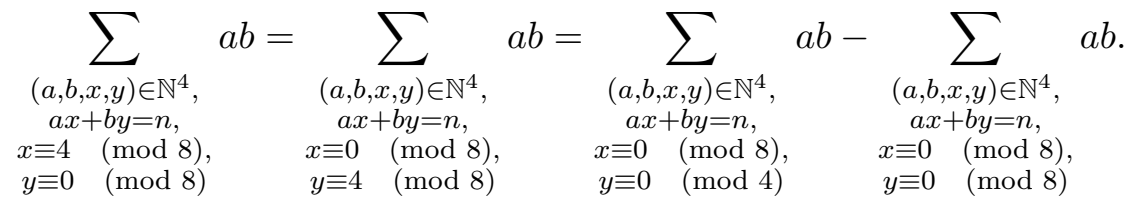

(iii) We subtract (ii) of Theorem 1.5 from (ii) of Theorem 1.4. That is,

$$
\sum_{\substack{(a, b, x, y) \in \mathbb{N}^{4}, a x+b y=n, x \equiv 4 \quad(\bmod 8),}} a b=\sum_{\substack{(a, b, x, y) \in \mathbb{N}^{4}, a x+b y=n, x \equiv 4 \quad(\bmod 8),}} a b-\sum_{\begin{array}{c}
(a, b, x, y) \in \mathbb{N}^{4}, \\
a x+b y=n, \\
y \equiv 4 \quad(\bmod 8)
\end{array}} a b .
$$


(iv) We have

$$
\begin{aligned}
& \sum_{\substack{(a, b, x, y) \in \mathbb{N}^{4}, a x+b y=n, x \neq 0(\bmod 8),}} a b=\sum_{k=1}^{n-1}\left(\sum_{\begin{array}{c}
a \mid k, \\
8 \nmid \frac{k}{a}
\end{array}} a\right)\left(\sum_{\begin{array}{c}
b \mid(n-k), \\
8 \nmid\left(\frac{n-k}{b}\right)
\end{array}} b\right) \\
& y \neq 0 \quad(\bmod 8) \\
& =\sum_{k=1}^{n-1}\left(\sum_{\substack{a|k \\
8| \frac{k}{a}}} a-\sum_{\substack{a|k, b|(n-k)}} b-\sum_{\substack{b|(n-k), 8|\left(\frac{n-k}{b}\right)}} b\right) \\
& =\sum_{k=1}^{n-1}\left(\sigma_{1}(k)-\sigma_{1}\left(\frac{k}{8}\right)\right)\left(\sigma_{1}(n-k)-\sigma_{1}\left(\frac{n-k}{8}\right)\right) \\
& =\sum_{k=1}^{n-1} \sigma_{1}(k) \sigma_{1}(n-k)-2 \sum_{k<\frac{n}{8}} \sigma_{1}(k) \sigma_{1}(n-8 k)+\sum_{k<\frac{n}{8}} \sigma_{1}(k) \sigma_{1}\left(\frac{n}{8}-k\right) \\
& =A_{1}(n)-2 A_{8}(n)+A_{1}\left(\frac{n}{8}\right) \text {. }
\end{aligned}
$$

Thus we use the formula for $A_{1}(n)$ and

$$
\begin{aligned}
A_{8}(n):= & \sum_{k<n / 8} \sigma_{1}(k) \sigma_{1}(n-8 k)=\frac{1}{192} \sigma_{3}(n)+\frac{1}{64} \sigma_{3}\left(\frac{n}{2}\right)+\frac{1}{16} \sigma_{3}\left(\frac{n}{4}\right) \\
& +\frac{1}{3} \sigma_{3}\left(\frac{n}{8}\right)+\left(\frac{1}{24}-\frac{n}{32}\right) \sigma_{1}(n)+\left(\frac{1}{24}-\frac{n}{4}\right) \sigma_{1}\left(\frac{n}{8}\right)-\frac{1}{64} c_{8}(n)
\end{aligned}
$$

from $[3,(1.1)]$.

(v) Note that

$$
\sum_{\begin{array}{c}
(a, b, x, y) \in \mathbb{N}^{4}, \\
a x+b y=n, \\
x=0 \quad(\bmod 8), \\
y \neq 0 \quad(\bmod 8)
\end{array}} a b=\frac{1}{2}\left\{A_{1}(n)-A_{1}\left(\frac{n}{8}\right)-\sum_{\begin{array}{r}
(a, b, x, y) \in \mathbb{N}^{4}, \\
a x+b y=n, \\
x \neq 0 \quad(\bmod 8), \\
y \neq 0 \quad(\bmod 8)
\end{array}} a b\right\},
$$

and hence the result follows from (iv) of Theorem 1.5 and the formula for $A_{1}(n)$. 


\section{Proof of the Theorem 1.6. :}

We subtract (iv) of Theorem 1.4 and (ii) of Theorem 1.5 from (v) of Theorem 1.5, since note that

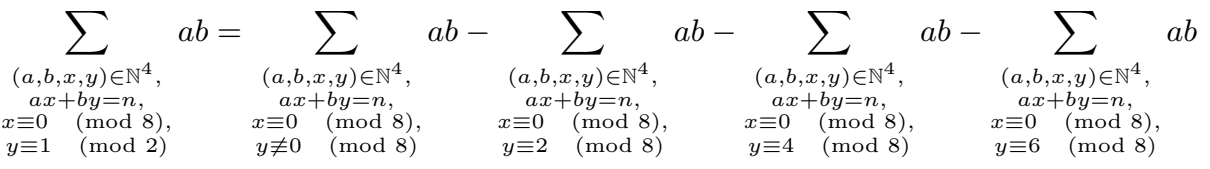

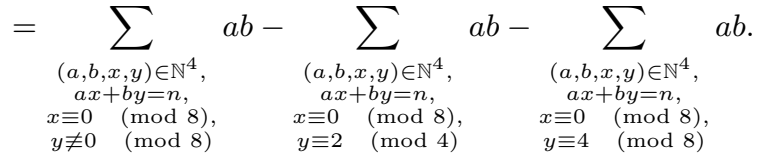

\section{Applications}

\subsection{Application to the Coefficient $c_{8}(n)$ :}

Lemma 4.1. If $n=2^{m} p_{1}^{e_{1}} \cdots p_{r}^{e_{r}}$ with $m=1$ or 2 and odd prime $p_{i}$, then we have

$$
\sigma_{3}^{*}(n) \equiv 2(3 n-4) \sigma_{1}^{*}(n) \quad(\bmod 192) .
$$

Proof. Since $m=1$ or 2 , and $n=2^{m} p_{1}^{e_{1}} \cdots p_{r}^{e_{r}}$, we note that $c_{8}(n)=0$ and $\sigma_{1}\left(\frac{n}{8}\right)=0$. Therefore from Corollary 1.6, we obtain

$$
192 \sum_{\substack{(a, b, x, y) \in \mathbb{N}^{4}, a x+b y=n, x \equiv 0 \\ y \equiv 1 \quad(\bmod 8), y \equiv 1 \quad(\bmod 2)}} a b=\sigma_{3}^{*}(n)-2(3 n-4) \sigma_{1}^{*}(n) .
$$

Thus the proof is complete.

Lemma 4.2. Let $n$ be an odd positive integer. Then we have

(i)

$$
c_{8}(n) \equiv-13 \sigma_{3}(n)+14 n \sigma_{1}(n) \quad(\bmod 64) .
$$

(ii)

$$
3 c_{8}(n) \equiv \sigma_{3}(n)-2(3 n-4) \sigma_{1}(n) \quad(\bmod 192) .
$$

(iii)

$$
c_{8}(n) \equiv \sigma_{1}(n) \quad(\bmod 8) .
$$

In particular, if $p_{i} \equiv-1(\bmod 8)$ is a prime with $p_{i}^{2 e_{i}-1} \mid n$ and $p_{i}^{2 e_{i}} \nmid n$, then $c_{8}(n) \equiv 0(\bmod 8)$. 
Proof. (i) For odd positive integer $n$, (iv) of Theorem 1.5 can be written as

$$
32 \sum_{k=1}^{n-1} \sigma_{1}^{*}(k ; 8) \sigma_{1}^{*}(n-k ; 8)=13 \sigma_{3}(n)-14 n \sigma_{1}(n)+c_{8}(n) .
$$

Then, since $\sigma_{1}^{*}(k) \sigma_{1}^{*}(n-k)=\sigma_{1}^{*}(n-k) \sigma_{1}^{*}(n-(n-k))$, we have

$$
64 \sum_{k=1}^{\frac{n-1}{2}} \sigma_{1}^{*}(k ; 8) \sigma_{1}^{*}(n-k ; 8)=13 \sigma_{3}(n)-14 n \sigma_{1}(n)+c_{8}(n) .
$$

Therefore $c_{8}(n) \equiv-13 \sigma_{3}(n)+14 n \sigma_{1}(n)(\bmod 64)$.

(ii) In a similar manner, (v) of Theorem 1.5 becomes

$$
192 \sum_{k=1}^{n-1} \sigma_{1}(k ; 8 ; 0) \sigma_{1}^{*}(n-k ; 8)=\sigma_{3}(n)-2(3 n-4) \sigma_{1}(n)-3 c_{8}(n) \text {. }
$$

Thus $3 c_{8}(n) \equiv \sigma_{3}(n)-2(3 n-4) \sigma_{1}(n)(\bmod 192)$.

(iii) Multiplying (ii) by 13 and subtracting (i), we obtain

$$
\begin{aligned}
& 64\left\{39 \sum_{k=1}^{n-1} \sigma_{1}(k ; 8 ; 0) \sigma_{1}^{*}(n-k ; 8)-\sum_{k=1}^{\frac{n-1}{2}} \sigma_{1}^{*}(k ; 8) \sigma_{1}^{*}(n-k ; 8)\right\} \\
& =(104-64 n) \sigma_{1}(n)-40 c_{8}(n) .
\end{aligned}
$$

(11) implies that

$$
5 c_{8}(n) \equiv 5 \sigma_{1}(n) \quad(\bmod 8) .
$$

Since $(5,8)=1$, we have $c_{8}(n) \equiv \sigma_{1}(n)(\bmod 8)$.

Remark 4.3. Glaisher [9, p. 300] and Ramanujan [29] proved that (12) $\sigma(1) \sigma(2 n-1)+\sigma(3) \sigma(2 n-3)+\cdots+\sigma(2 n-1) \sigma(1)=\frac{1}{8}\left[\sigma_{3}(2 n)-\sigma_{3}(n)\right]$.

We note that

$$
\sigma_{s}^{*}(n)=\sigma_{s}(n)-\sigma_{s}\left(\frac{n}{2}\right)
$$

(see [31, Theorem 3.3]). Thus (12) becomes

$$
\begin{aligned}
& \sigma(1) \sigma(2 n-1)+\sigma(3) \sigma(2 n-3)+\cdots+\sigma(2 n-1) \sigma(1) \\
& =\frac{1}{8} \sigma_{3}^{*}(2 n)=\frac{1}{8} \sum_{\substack{d \in \mathbb{N}, d \mid 2 n, \frac{2 n}{d} \text { odd }}} d^{3}=\frac{1}{8} \sum_{\substack{e \in \mathbb{N}, e \mid n, \frac{n}{e} \text { odd }}}(2 e)^{3}=\frac{1}{8} \cdot 8 \sum_{\substack{e \in \mathbb{N}, e \mid n, \frac{n}{e} \text { odd }}} e^{3}=\sigma_{3}^{*}(n)
\end{aligned}
$$


by letting $e=\frac{d}{2}$. From (iii) of Lemma 4.2 we have

(13) $c_{8}(1) c_{8}(2 n-1)+c_{8}(3) c_{8}(2 n-3)+\cdots+c_{8}(2 n-1) c_{8}(1) \equiv \sigma_{3}^{*}(n) \quad(\bmod 8)$.

\subsection{Application Involving the Coefficient $D(n)$ :}

Theorem 4.4. Let $\sum_{n=1}^{\infty} D(n) q^{n}=q^{2} \prod_{m=1}^{\infty}\left(1-q^{2 m}\right)^{8}\left(1-q^{4 m}\right)^{8}$. Then we have

(i)

$$
D(2 n) \equiv \sigma_{3}^{*}(n) \quad(\bmod 8)
$$

(ii)

$$
\sum_{k=1}^{2 n} c_{8}(k) c_{8}(2 n+1-k)=0 .
$$

Proof. (i) Since $c_{8}(2 k)=0$, we have

$$
\begin{aligned}
D(2 n) & =\sum_{k=1}^{2 n-1} c_{8}(k) c_{8}(2 n-k) \\
& =c_{8}(1) c_{8}(2 n-1)+c_{8}(3) c_{8}(2 n-3)+\cdots+c_{8}(2 n-1) c_{8}(1) \\
& \equiv \sigma_{3}^{*}(n) \quad(\bmod 8)
\end{aligned}
$$

by (13).

(ii) We note that if $k$ is even, then $c_{8}(k)=0$ and if $k$ is odd then $c_{8}(2 n+1-k)=0$. Therefore $c_{8}(k) c_{8}(2 n+1-k)=0$ for all $k$ satisfying $1 \leq k \leq 2 n$. Hence

$$
\sum_{k=1}^{2 n} c_{8}(k) c_{8}(2 n+1-k)=0 .
$$

Let $\Lambda_{\tau}=\mathbb{Z}+\tau \mathbb{Z}(\tau \in \mathcal{H}$ the complex upper half plane) be a lattice and $z \in \mathbb{C}$. The Weierstrass $\wp$ function related to $\Lambda_{\tau}$ is defined by the series

$$
\wp\left(z ; \Lambda_{\tau}\right)=\frac{1}{z^{2}}+\sum_{\substack{\omega \in \Lambda_{\tau} \\ \omega \neq 0}}\left\{\frac{1}{(z-\omega)^{2}}-\frac{1}{\omega^{2}}\right\},
$$

and the Eisenstein series of weight $2 k$ for $\Lambda_{\tau}$ for any integer $k \geq 2$ is given by the series

$$
G_{2 k}\left(\Lambda_{\tau}\right)=\sum_{\substack{\omega \in \Lambda_{\tau} \\ \omega \neq 0}} \omega^{-2 k}
$$


We use the notations $\wp(z)$ and $G_{2 k}$ instead of $\wp\left(z ; \Lambda_{\tau}\right)$ and $G_{2 k}\left(\Lambda_{\tau}\right)$, respectively, when the lattice $\Lambda_{\tau}$ has been fixed. Now, Laurent series for $\wp(z)$ about $z=0$ is given by

$$
\wp(z)=z^{-2}+\sum_{k=1}^{\infty}(2 k+1) G_{2 k+2} z^{2 k} .
$$

As is customary, by setting

$$
g_{2}(\tau)=g_{2}\left(\Lambda_{\tau}\right)=60 G_{4} \quad \text { and } \quad g_{3}(\tau)=g_{3}\left(\Lambda_{\tau}\right)=140 G_{6},
$$

the algebraic relation between $\wp(z)$ and $\wp^{\prime}(z)$ becomes

$$
\wp^{\prime}(z)^{2}=4 \wp(z)^{3}-g_{2}(\tau) \wp(z)-g_{3}(\tau) .
$$

Corollary 4.5. Let $\chi(n)=\left\{\begin{array}{ll}0, & \text { for odd } n, \\ 1, & \text { for even } n .\end{array}\right.$ Then we have

$$
\sum_{n=1}^{\infty} \chi(n) D(n) q^{\frac{n}{2}} \equiv \frac{\wp^{\prime \prime}\left(\frac{\tau}{2}, \tau\right)}{32 \pi^{4}} \quad(\bmod 8) .
$$

Proof. From [17, (28)], we see that

$$
\begin{aligned}
\wp^{\prime \prime}\left(\frac{\tau}{2}, \tau\right) & =32 \pi^{4} q \frac{\left(q^{2} ; q^{2}\right)_{\infty}^{16}}{(q)_{\infty}^{8}} \\
& =32 \pi^{4}\left(\sum_{n=1}^{\infty} \sigma_{3}(n) q^{n}-\sum_{n=1}^{\infty} \sigma_{3}(n) q^{2 n}\right) \\
& =32 \pi^{4} \sum_{n=1}^{\infty} \sigma_{3}^{*}(n) q^{n} .
\end{aligned}
$$

Therefore, from (i) of Theorem 4.4, we obtain

$$
\frac{\wp^{\prime \prime}\left(\frac{\tau}{2}, \tau\right)}{32 \pi^{4}}=q \frac{\left(q^{2} ; q^{2}\right)_{\infty}^{16}}{(q)_{\infty}^{8}}=\sum_{n=1}^{\infty} \sigma_{3}^{*}(n) q^{n} \equiv \sum_{n=1}^{\infty} \chi(n) D(n) q^{\frac{n}{2}} \quad(\bmod 8)
$$

\subsection{Application to the Function $r_{8}(4 n)$ :}

The $s$ squares problem is to count the number $r_{s}(n)$ of integer solutions $\left(x_{1}, \cdots, x_{s}\right)$ of the Diophantine equation

$$
x_{1}^{2}+\cdots+x_{s}^{2}=n,
$$

where changing the sign of the order of the $x_{i}^{\prime} s$ gives distinct solutions. Using the theory of theta functions, Jacobi studied the number $r_{s}(n)$ 
of integer solutions of Eq. (15) for some important cases. In fact, he considered the equation

$$
\vartheta_{3}(0,-q)^{s}:=1+\sum_{n=1}^{\infty}(-1)^{n} r_{s}(n) q^{n},
$$

where

$$
\vartheta_{3}(z, q):=1+2 \sum_{n=1}^{\infty} q^{n^{2}} \cos (2 n z)
$$

(See [30, p. 258]) and thus

$$
\vartheta_{3}(0, q)=\sum_{j=-\infty}^{\infty} q^{j^{2}} .
$$

Jacobi [15] also established that

$$
\begin{aligned}
\vartheta_{3}(0,-q)^{4} & =1-8 \sum_{r=1}^{\infty}(-1)^{r-1} \frac{r q^{r}}{1+q^{r}} \\
& =1+8 \sum_{n=1}^{\infty}(-1)^{n}\left(\sum_{\substack{d \mid n, d>0, 4 \nmid d}} d\right) q^{n} .
\end{aligned}
$$

Remark 4.6. From (16) and (17) we see that

$$
r_{4}(n)=8 \sum_{\substack{d \mid n, 4 \nmid d}} d=8\left\{\sum_{\substack{d|n \\ 4| d}} d-\sum_{\substack{d|n, 4| d}} d\right\}=8\left\{\sigma_{1}(n)-4 \sigma_{1}\left(\frac{n}{4}\right)\right\} .
$$

Theorem 4.7. For $n \in \mathbb{N}$, we have

$$
r_{8}(4 n)=16\left\{56 \sigma_{3}^{*}(n)+15 \sigma_{3}(n)\right\} .
$$

Proof. By the property of $r_{8}(n)$ and (18), we can write

(19)

$$
\begin{aligned}
r_{8}(4 n)= & \sum_{k=0}^{4 n} r_{4}(k) r_{4}(4 n-k) \\
= & 2 r_{4}(4 n)+\sum_{k=1}^{4 n-1} r_{4}(k) r_{4}(4 n-k) \\
= & 16\left\{\sigma_{1}(4 n)-4 \sigma_{1}(n)\right\} \\
& \quad+64 \sum_{k=1}^{4 n-1}\left\{\sigma_{1}(k)-4 \sigma_{1}\left(\frac{k}{4}\right)\right\}\left\{\sigma_{1}(4 n-k)-4 \sigma_{1}\left(\frac{4 n-k}{4}\right)\right\} .
\end{aligned}
$$


Note that

$$
\begin{aligned}
& \sum_{k=1}^{4 n-1}\left\{\sigma_{1}(k)-4 \sigma_{1}\left(\frac{k}{4}\right)\right\}\left\{\sigma_{1}(4 n-k)-4 \sigma_{1}\left(\frac{4 n-k}{4}\right)\right\} \\
& =4 \sum_{\begin{array}{c}
(a, b, x, y) \in \mathbb{N}^{4}, \\
a x+b y=n, \\
x \neq 0(\bmod 4), \\
y \neq 0 \quad(\bmod 4)
\end{array}} a b-3 \sum_{k=1}^{4 n-1} \sigma_{1}(k) \sigma_{1}(4 n-k)+12 \sum_{k=1}^{n-1} \sigma_{1}(k) \sigma_{1}(n-k) \\
& =4 \sum_{\begin{array}{c}
(a, b, x, y) \in \mathbb{N}^{4}, \\
a x+b y=n, \\
x \neq 0 \quad(\bmod 4), \\
y \neq 0 \quad(\bmod 4)
\end{array}} a b-3 A_{1}(4 n)+12 A_{1}(n) \\
& =\frac{1}{4}\left\{\sigma_{3}(4 n)-2 \sigma_{3}(2 n)+16 \sigma_{3}(n)-\sigma_{1}(4 n)+4 \sigma_{1}(n)\right\} .
\end{aligned}
$$

Note that we use $A_{1}(n)$ as in Section 1 and we also use $\sigma_{i}^{*}(n)=\sigma_{i}(n)-$ $\sigma_{i}\left(\frac{n}{2}\right)$ along with (iv) of Theorem 1.2. Finally, to replace $4 n$ and $2 n$ into $n$ in the last step, we use the equation $\sigma_{k}(p n)-\left(p^{k}+1\right) \sigma_{k}(n)+p^{k} \sigma_{k}\left(\frac{n}{p}\right)=0$ for any prime $p$ and $k, n \in \mathbb{N}$.

\subsection{Explicit Formulae for several Twisted Convolution Sums :}

Theorem 4.8. Let $n \in \mathbb{N}$ and let $\sigma_{1}(n ; u ; r):=\sum_{\frac{n}{d} \equiv r(\bmod u)} d$ where $u$ is the modulo and $r$ is residue of modulo $u$. Note that $\sigma_{1}(n ; 2 ; 1)=$ $\sigma_{1}^{*}(n ; 2)=\sigma_{1}^{*}(n)$. Then, we have 


\begin{tabular}{|c|c|}
\hline Convolution sums & Convolution formula \\
\hline$\sum_{k=1}^{n-1} \sigma_{1}(k ; 2 ; 0) \sigma_{1}(n-k ; 2 ; 0)$ & $\frac{1}{12}\left(5 \sigma_{3}\left(\frac{n}{2}\right)+(1-3 n) \sigma_{1}\left(\frac{n}{2}\right)\right)$ \\
\hline$\sum_{k=1}^{n-1} \sigma_{1}^{*}(k ; 2) \sigma_{1}(n-k ; 2 ; 0)$ & $\frac{1}{24}\left(2 \sigma_{3}^{*}(n)+\sigma_{1}^{*}(n)-3 n \sigma_{1}(n)\right)$ \\
\hline$\sum_{k=1}^{n-1} \sigma_{1}^{*}(k ; 2) \sigma_{1}^{*}(n-k)$ & $\frac{1}{4}\left(\sigma_{3}^{*}(n)-n \sigma_{1}^{*}(n)\right)$ \\
\hline$\sum_{k=1}^{n-1} \sigma_{1}(k ; 4 ; 0) \sigma_{1}(n-k ; 4 ; 0)$ & $\frac{1}{12}\left(5 \sigma_{3}\left(\frac{n}{4}\right)+\left(1-\frac{3}{2} n\right) \sigma_{1}\left(\frac{n}{4}\right)\right)$ \\
\hline$\sum_{k=1}^{n-1} \sigma_{1}(k ; 4 ; 2) \sigma_{1}(n-k ; 4 ; 2)$ & $\frac{1}{8}\left(2 \sigma_{3}^{*}\left(\frac{n}{2}\right)-n \sigma_{1}^{*}\left(\frac{n}{2}\right)\right)$ \\
\hline$\sum_{k=1}^{n-1} \sigma_{1}(k ; 4 ; 0) \sigma_{1}(n-k ; 4 ; 2)$ & $\frac{1}{24}\left\{2 \sigma_{3}^{*}\left(\frac{n}{2}\right)+\sigma_{1}^{*}\left(\frac{n}{2}\right)-\frac{3}{2} n \sigma_{1}\left(\frac{n}{2}\right)\right\}$ \\
\hline$\sum_{k=1}^{n-1} \sigma_{1}^{*}(k ; 4) \sigma_{1}^{*}(n-k ; 4)$ & $\frac{1}{8}\left[3 \sigma_{3}^{*}(n)+2 \sigma_{3}^{*}\left(\frac{n}{2}\right)-3 n\left\{\sigma_{1}^{*}(n)+\sigma_{1}^{*}\left(\frac{n}{2}\right)\right\}\right]$ \\
\hline$\sum_{k=1}^{n-1} \sigma_{1}(k ; 4 ; 0) \sigma_{1}^{*}(n-k ; 4)$ & $\begin{array}{c}\frac{1}{48}\left[\sigma_{3}^{*}(n)+4 \sigma_{3}^{*}\left(\frac{n}{2}\right)\right. \\
\left.-(3 n-2)\left\{\sigma_{1}^{*}(n)+\sigma_{1}^{*}\left(\frac{n}{2}\right)\right\}-9 n \sigma_{1}\left(\frac{n}{4}\right)\right]\end{array}$ \\
\hline$\sum_{k=1}^{n-1} \sigma_{1}(k ; 4 ; 0) \sigma_{1}^{*}(n-k)$ & $\frac{1}{48}\left[\sigma_{3}^{*}(n)-(3 n-2) \sigma_{1}^{*}(n)-6 n \sigma_{1}\left(\frac{n}{4}\right)\right]$ \\
\hline$\sum_{k=1}^{n-1} \sigma_{1}(k ; 2 ; 0) \sigma_{1}(n-k ; 4 ; 2)$ & $\frac{1}{48}\left(16 \sigma_{3}^{*}\left(\frac{n}{2}\right)-2(3 n-1) \sigma_{1}^{*}\left(\frac{n}{2}\right)-3 n \sigma_{1}\left(\frac{n}{2}\right)\right)$ \\
\hline$\sum_{k=1}^{n-1} \sigma_{1}(k ; 4 ; 0) \sigma_{1}(n-k ; 2 ; 0)$ & $\begin{aligned} \frac{1}{24}\left(2 \sigma_{3}\left(\frac{n}{2}\right)\right. & +8 \sigma_{3}\left(\frac{n}{4}\right)+\frac{1}{2}(2-3 n) \sigma_{1}\left(\frac{n}{2}\right) \\
& \left.+(1-3 n) \sigma_{1}\left(\frac{n}{4}\right)\right)\end{aligned}$ \\
\hline$\sum_{k=1}^{n-1} \sigma_{1}(k ; 8 ; 0) \sigma_{1}(n-k ; 4 ; 0)$ & $\begin{array}{c}\frac{1}{96}\left[8\left\{\sigma_{3}\left(\frac{n}{4}\right)+4 \sigma_{3}\left(\frac{n}{8}\right)\right\}-(3 n-4) \sigma_{1}\left(\frac{n}{4}\right)\right. \\
\left.-2(3 n-2) \sigma_{1}\left(\frac{n}{8}\right)\right]\end{array}$ \\
\hline$\sum_{k=1}^{n-1} \sigma_{1}(k ; 8 ; 4) \sigma_{1}(n-k ; 4 ; 0)$ & $\frac{1}{96}\left\{32 \sigma_{3}^{*}\left(\frac{n}{4}\right)-(9 n-4) \sigma_{1}^{*}\left(\frac{n}{4}\right)-3 n \sigma_{1}\left(\frac{n}{8}\right)\right\}$ \\
\hline$\sum_{k=1}^{n-1} \sigma_{1}(k ; 8 ; 0) \sigma_{1}(n-k ; 2 ; 0)$ & $\begin{array}{c}\frac{1}{48}\left(\sigma_{3}\left(\frac{n}{2}\right)+\left(2-\frac{3 n}{2}\right) \sigma_{1}\left(\frac{n}{2}\right)+3 \sigma_{3}\left(\frac{n}{4}\right)\right. \\
\left.+16 \sigma_{3}\left(\frac{n}{8}\right)+(2-6 n) \sigma_{1}\left(\frac{n}{8}\right)\right)\end{array}$ \\
\hline$\sum_{k=1}^{n-1} \sigma_{1}(k ; 8 ; 0) \sigma_{1}(n-k ; 4 ; 2)$ & $\frac{1}{96}\left\{2 \sigma_{3}^{*}\left(\frac{n}{2}\right)-(3 n-4) \sigma_{1}^{*}\left(\frac{n}{2}\right)-6 n \sigma_{1}\left(\frac{n}{8}\right)\right\}$ \\
\hline$\sum_{k=1}^{n-1} \sigma_{1}(k ; 8 ; 4) \sigma_{1}(n-k ; 2 ; 0)$ & $\begin{array}{c}\frac{1}{96}\left\{6 \sigma_{3}^{*}\left(\frac{n}{2}\right)+32 \sigma_{3}^{*}\left(\frac{n}{4}\right)\right. \\
\left.-3 n \sigma_{1}\left(\frac{n}{2}\right)-4(3 n-1) \sigma_{1}^{*}\left(\frac{n}{4}\right)\right\} \\
\end{array}$ \\
\hline$\sum_{k=1}^{n-1} \sigma_{1}(k ; 8 ; 4) \sigma_{1}(n-k ; 4 ; 2)$ & $\frac{1}{32}\left\{2 \sigma_{3}^{*}\left(\frac{n}{2}\right)-n \sigma_{1}^{*}\left(\frac{n}{2}\right)-2 n \sigma_{1}^{*}\left(\frac{n}{4}\right)\right\}$ \\
\hline$\sum_{k=1}^{n-1} \sigma_{1}(k ; 8 ; 0) \sigma_{1}(n-k ; 8 ; 0)$ & $\frac{1}{48}\left\{20 \sigma_{3}\left(\frac{n}{8}\right)-(3 n-4) \sigma_{1}\left(\frac{n}{8}\right)\right\}$ \\
\hline$\sum_{k=1}^{n-1} \sigma_{1}(k ; 8 ; 4) \sigma_{1}(n-k ; 8 ; 0)$ & $\frac{1}{96}\left\{8 \sigma_{3}^{*}\left(\frac{n}{4}\right)+4 \sigma_{1}^{*}\left(\frac{n}{4}\right)-3 n \sigma_{1}\left(\frac{n}{4}\right)\right\}$ \\
\hline$\sum_{k=1}^{n-1} \sigma_{1}(k ; 8 ; 4) \sigma_{1}(n-k ; 8 ; 4)$ & $\frac{1}{16}\left\{4 \sigma_{3}^{*}\left(\frac{n}{4}\right)-n \sigma_{1}^{*}\left(\frac{n}{4}\right)\right\}$ \\
\hline$\sum_{k=1}^{n-1} \sigma_{1}^{*}(k ; 8) \sigma_{1}^{*}(n-k ; 8)$ & $\begin{array}{c}\frac{1}{32}\left[13 \sigma_{3}^{*}(n)+12 \sigma_{3}^{*}\left(\frac{n}{2}\right)+8 \sigma_{3}^{*}\left(\frac{n}{4}\right)\right. \\
\left.-14 n\left\{\sigma_{1}^{*}(n)+\sigma_{1}^{*}\left(\frac{n}{2}\right)+\sigma_{1}^{*}\left(\frac{n}{4}\right)\right\}+c_{8}(n)\right]\end{array}$ \\
\hline$\sum_{k=1}^{n-1} \sigma_{1}(k ; 8 ; 0) \sigma_{1}^{*}(n-k ; 8)$ & $\begin{array}{c}\frac{1}{192}\left\{\sigma_{3}^{*}(n)+4 \sigma_{3}^{*}\left(\frac{n}{2}\right)+16 \sigma_{3}^{*}\left(\frac{n}{4}\right)-2(3 n-4) \sigma_{1}(n)\right. \\
\left.-4(9 n+2) \sigma_{1}\left(\frac{n}{8}\right)-3 c_{8}(n)\right\}\end{array}$ \\
\hline$\sum_{k=1}^{n-1} \sigma_{1}(k ; 8 ; 0) \sigma_{1}^{*}(n-k)$ & $\frac{1}{192}\left\{\sigma_{3}^{*}(n)-2(3 n-4) \sigma_{1}^{*}(n)-24 n \sigma_{1}\left(\frac{n}{8}\right)-3 c_{8}(n)\right\}$ \\
\hline
\end{tabular}

TABLE 5. Convolution sums

Proof. The proof follows from Theorems 1.1-1.6.

Corollary 4.9. For $n \in \mathbb{N}$, we have

$$
\sigma_{3}^{*}(n) \equiv(3 n-2) \sigma_{1}^{*}(n) \quad(\bmod 24) .
$$

In particular, if $n \not \equiv 0(\bmod 4)$, then $\sigma_{3}^{*}(n) \equiv(3 n-2) \sigma_{1}^{*}(n)(\bmod 48)$.

Proof. From Table 5, we observe that

$$
\sum_{k=1}^{n-1} \sigma_{1}(k ; 4 ; 0) \sigma_{1}^{*}(n-k)=\frac{1}{48}\left[\sigma_{3}^{*}(n)-(3 n-2) \sigma_{1}^{*}(n)-6 n \sigma_{1}\left(\frac{n}{4}\right)\right] \text {. }
$$


If $n \equiv 0(\bmod 4)$, i.e., $n=4 l$, then we have

$$
48 \sum_{k=1}^{4 l-1} \sigma_{1}(k ; 4 ; 0) \sigma_{1}^{*}(4 l-k)=\sigma_{3}^{*}(4 l)-(12 l-2) \sigma_{1}^{*}(4 l)-24 l \sigma_{1}(l) .
$$

Thus $\sigma_{3}^{*}(n) \equiv(3 n-2) \sigma_{1}^{*}(n)(\bmod 24)$. If $n \not \equiv 0(\bmod 4)$ then we obtain

$$
48 \sum_{k=1}^{n-1} \sigma_{1}(k ; 4 ; 0) \sigma_{1}^{*}(n-k)=\sigma_{3}^{*}(n)-(3 n-2) \sigma_{1}^{*}(n) .
$$

So $\sigma_{3}^{*}(n) \equiv(3 n-2) \sigma_{1}^{*}(n)(\bmod 48)$.

\subsection{Convolution Sums and Congruences Involving the Arith- metic Function $c(n)$ :}

We denote by $\mathcal{H}=\{\tau \in \mathbb{C} \mid \operatorname{Im}(\tau)>0\}$ the complex upper half-plane and for $\tau \in \mathcal{H}$ write $q=e^{2 \pi i \tau}$ and $Y=4 \pi \operatorname{Im}(\tau)$, while $D$ denotes the differential operator $D=\frac{1}{2 \pi i} \frac{d}{d \tau}=q \frac{d}{d q}$. Recall that a modular form of weight $k$ on a subgroup $\Gamma$ of finite index of $\Gamma_{1}$ is a holomorphic function $f$ on $\mathcal{H}$ satisfying

$$
f\left(\frac{a \tau+b}{c \tau+d}\right)=(c \tau+d)^{k} f(\tau), \quad \forall \tau \in \mathcal{H}, \quad\left(\begin{array}{ll}
a & b \\
c & d
\end{array}\right) \in \Gamma
$$

and growing at most polynomially in $1 / Y$ as $Y \rightarrow 0$. If $\left(\begin{array}{ll}1 & \lambda \\ 0 & 1\end{array}\right) \in \Gamma$, then these conditions imply that $f$ has a convergent Fourier series expansion namely, $f(\tau)=\sum_{n=0}^{\infty} a(n) q^{n / \lambda}$ at infinity. The space of holomorphic modular forms of weight $k$ on $\Gamma$ is denoted by $M_{k}(\Gamma)$ and the graded ring $\oplus_{k} M_{k}(\Gamma)$ by $M_{*}(\Gamma)$.

Resembling the holomorphic modular forms, there are also functions $F(\tau)$ which satisfy the same transformation properties and growth conditions as before but which belong to $\mathbb{C}\left[\left[q^{1 / \lambda}\right]\right]\left[Y^{-1}\right]$ instead of $\mathbb{C}\left[\left[q^{1 / \lambda}\right]\right]$, i.e. which have the form

$$
F(\tau)=\sum_{m=0}^{M} f_{m}(\tau) Y^{-m} \quad\left(f_{m}(\tau) \text { holomorphic for } m=0,1, \ldots, M\right)
$$

for some integer $M \geq 0$ (and necessarily $\leq k / 2$ ). We call such a function an almost-holomorphic modular form of weight $k$ and denote the vector space of them by $\widehat{M}_{k}(\Gamma)$, while the holomorphic function $f_{0}(\tau)$ obtained formally as the "constant term with respect to $1 / Y$ " of $f$ will 
be called a quasi-modular form of weight $k$ and the space of such functions denoted by $\widetilde{M}_{k}(\Gamma)$. It is clear that the spaces $\widehat{M}_{*}(\Gamma)=\oplus \widehat{M}_{k}(\Gamma)$ and $\widetilde{M}_{*}(\Gamma)=\oplus \widetilde{M}_{k}(\Gamma)$ are graded rings and the map $\widetilde{M}_{*}(\Gamma) \rightarrow \widetilde{M}_{*}(\Gamma)$ is a ring homomorphism (see [16]).

Let $\widehat{M}_{*}\left(\Gamma_{1}\right)$ denote the graded ring of quasimodular forms on the full modular group $\Gamma_{1}=P S L(2, \mathbb{Z})$. This is the ring generated by $G_{2}, G_{4}, G_{6}$ and graded by assigning to each $G_{k}$ the weight $k$, where

$$
\begin{aligned}
& G_{k}(\tau)=-\frac{B_{k}}{2 k}+\sum_{n=1}^{\infty}\left(\sum_{d \mid n} d^{k-1}\right) q^{n}, \\
& \left(k=2,4,6, \ldots, \quad B_{k}=\text { k-th Bernoulli numbers }\right),
\end{aligned}
$$

and let

$$
G_{k}^{*}(\tau)=G_{k}(\tau)-G_{k}(2 \tau)
$$

are the Eisenstein series, all of which except $G_{2}$ are modular. If we consider the coefficient of $q^{n}$ in $G_{k}$ and $G_{k}^{*}$, then we determine the values of $\sigma_{k-1}(n)$.

Using the theory of quasi-modular forms stated above, we get two identities below in Lemma 4.11 of convolution sums involving divisor functions.

Proposition 4.10. Let $\Gamma \subset S L_{2}(\mathbb{Z})$ be a subgroup of finite index of the full modular group. Let $\widetilde{M}_{k}(\Gamma)$ be the space of quasi modular forms of weight $k$ on $\Gamma$. Then

(1) For $f_{1} \in \widetilde{M}_{k_{1}}(\Gamma)$ and $f_{2} \in \widetilde{M}_{k_{2}}(\Gamma), f_{1} f_{2} \in \widetilde{M}_{k_{1}+k_{2}}(\Gamma)$.

(2) For even $k>0, \widetilde{M}_{k}(\Gamma)=\oplus_{0 \leq i \leq k / 2} D^{i} M_{k-2 i}(\Gamma) \oplus<D^{k / 2-1} G_{2}>$.

Proof. The first assertion is easy and for second one see Proposition 1 in $[16]$.

Lemma 4.11. For any integer $n \geq 3$, we have

(i)

$$
\begin{array}{r}
\sum_{1 \leq k<n / 2} k^{2} \sigma_{1}(k) \sigma_{1}(n-2 k)=\frac{1}{960}\left\{6 n^{2} \sigma_{3}(n)+24 n^{2} \sigma_{3}\left(\frac{n}{2}\right)-5 n^{3} \sigma_{1}(n)\right. \\
\left.-30 n^{3} \sigma_{1}\left(\frac{n}{2}\right)+10 n^{2} \sigma_{1}\left(\frac{n}{2}\right)-c(n)\right\}
\end{array}
$$

where

$$
\sum_{n=1}^{\infty} c(n) q^{n}:=q \prod_{m=1}^{\infty}\left(1-q^{m}\right)^{8}\left(1-q^{2 m}\right)^{8}
$$


and

(ii)

$$
\begin{aligned}
& \sum_{1 \leq k<n / 2} k \sigma_{1}(k) \sigma_{1}(n-2 k) \\
= & \frac{1}{48} n\left\{\sigma_{3}(n)+4 \sigma_{3}\left(\frac{n}{2}\right)-n \sigma_{1}(n)-4 n \sigma_{1}\left(\frac{n}{2}\right)+\sigma_{1}\left(\frac{n}{2}\right)\right\} .
\end{aligned}
$$

Proof. (i) Let

$$
\eta(\tau):=q^{\frac{1}{24}} \prod_{m=1}^{\infty}\left(1-q^{m}\right)
$$

with $q=e^{2 \pi i \tau}, \tau \in \mathcal{H}$ and

$\Gamma_{0}(2):=\left\{\left(\begin{array}{ll}a & b \\ c & d\end{array}\right) \in \Gamma(1): c \equiv 0 \quad(\bmod 2)\right\}, \Gamma(1):=S L(2, \mathbb{Z}) /\{ \pm 1\}$.

We note that

$$
\eta^{8}(\tau) \eta^{8}(2 \tau)=q \prod_{m=1}^{\infty}\left(1-q^{m}\right)^{8}\left(1-q^{2 m}\right)^{8}=\sum_{n=1}^{\infty} c(n) q^{n}
$$

is a unique normalized newform in $S_{8}\left(\Gamma_{0}(2)\right)$ (the space of cusp forms of weight 8 associated to the congruence group $\left.\Gamma_{0}(2)\right)$. So, we take $\Gamma=\Gamma_{0}(2)$ in Proposition 4.10 and observe that with $k=8$,

$$
\begin{aligned}
\widetilde{M}_{8}\left(\Gamma_{0}(2)\right) & =M_{8}\left(\Gamma_{0}(2)\right) \oplus D M_{6}\left(\Gamma_{0}(2)\right) \oplus D^{2} M_{4}\left(\Gamma_{0}(2)\right) \\
& \oplus D^{3} M_{2}\left(\Gamma_{0}(2)\right) \oplus<D^{3} G_{2}>
\end{aligned}
$$

since $M_{0}\left(\Gamma_{0}(2)\right)=\{0\}$. We also note that with $k_{1}=6$ and $k_{2}=2$ in the Proposition 4.10, the functions

$$
f_{1}(\tau)=D^{2} G_{2}(2 \tau) \in<D^{3-1} G_{2}(2 \tau)>\subset \widetilde{M}_{6}\left(\Gamma_{0}(2)\right)
$$

and

$$
f_{2}(\tau)=G_{2}(\tau) \in<G_{2}(\tau)>\subset \widetilde{M}_{2}\left(\Gamma_{0}(2)\right)
$$

are quasi-modular forms and the product

$$
f_{1}(\tau) f_{2}(\tau)=\left(4 \sum_{m=1}^{\infty} m^{2} \sigma_{1}(m) q^{2 m}\right)\left(-\frac{1}{24}+\sum_{n=1}^{\infty} \sigma_{1}(n) q^{n}\right) \in \widetilde{M}_{8}\left(\Gamma_{0}(2)\right) .
$$


Hence there exist a unique tuplet $\left(a_{1}, a_{2}, \cdots, a_{11}\right)$ of rational numbers $a_{j}$ such that

$$
\begin{aligned}
& \sum_{1 \leq k<n / 2} k^{2} \sigma_{1}(k) \sigma_{1}(n-2 k)=a_{1} n^{2} \sigma_{3}(n)+a_{2} n^{2} \sigma_{3}\left(\frac{n}{2}\right)+a_{3} n^{3} \sigma_{1}(n) \\
& \quad+a_{4} n^{3} \sigma_{1}\left(\frac{n}{2}\right)+a_{5} n^{2} \sigma_{1}(n)+a_{6} n^{2} \sigma_{1}\left(\frac{n}{2}\right)+a_{7} n \sigma_{1}(n) \\
& \quad+a_{8} n \sigma_{1}\left(\frac{n}{2}\right)+a_{9} \sigma_{1}(n)+a_{10} \sigma_{1}\left(\frac{n}{2}\right)+a_{11} c(n)
\end{aligned}
$$

holds for every integer $n \geq 1$. Therefore, we get 11 equations in 11 variables by taking $n=1, \cdots, 11$. Using Mathematica 8.0, we solve these simultaneous equations to obtain

$$
\begin{aligned}
& a_{1}=\frac{1}{160}, \quad a_{2}=\frac{1}{40}, \quad a_{3}=-\frac{1}{192}, \quad a_{4}=-\frac{1}{32}, \quad a_{5}=0, \\
& a_{6}=\frac{1}{96}, \quad a_{7}=0, \quad a_{8}=0, \quad a_{9}=0, \quad a_{10}=0, \quad a_{11}=-\frac{1}{960} .
\end{aligned}
$$

Therefore,

$$
\begin{aligned}
\sum_{1 \leq k<n / 2} k^{2} \sigma_{1}(k) \sigma_{1}(n-2 k)= & \frac{1}{160} n^{2} \sigma_{3}(n)+\frac{1}{40} n^{2} \sigma_{3}\left(\frac{n}{2}\right)-\frac{1}{192} n^{3} \sigma_{1}(n) \\
& -\frac{1}{32} n^{3} \sigma_{1}\left(\frac{n}{2}\right)+\frac{1}{96} n^{2} \sigma_{1}\left(\frac{n}{2}\right)-\frac{1}{960} c(n) .
\end{aligned}
$$

(ii) We take $\Gamma=\Gamma_{0}(2), k_{1}=k_{2}=4, k=k_{1}+k_{2}=8$ in Proposition 4.10. We note that

$$
\begin{aligned}
& f_{1}(\tau)=D G_{2}(2 \tau) \in<D^{2-1} G_{2}(2 \tau)>\subset \widetilde{M}_{4}\left(\Gamma_{0}(2)\right), \\
& f_{2}(\tau)=G_{4}(\tau) \in<M_{4}\left(\Gamma_{0}(2)\right)>\subset \widetilde{M}_{4}\left(\Gamma_{0}(2)\right)
\end{aligned}
$$

with $f_{1} f_{2} \in \widetilde{M}_{8}\left(\Gamma_{0}(2)\right)$ by Proposition 4.10 . We also note that

$$
f_{1}(\tau) f_{2}(\tau)=2\left(\sum_{m=1}^{\infty} m \sigma_{1}(m) q^{2 m}\right)\left(\frac{1}{240}+\sum_{n=1}^{\infty} \sigma_{3}(n) q^{n}\right) \in \widetilde{M}_{8}\left(\Gamma_{0}(2)\right) .
$$

Now the proof of (ii) follows in a similar fashion as of (i).

Lemma 4.12. For any integer $n \geq 2$, we have

$$
\sum_{k=1}^{n-1} k^{2} \sigma_{1}^{*}(k) \sigma_{1}^{*}(n-k)=\frac{1}{120}\left\{9 n^{2} \sigma_{3}^{*}(n)-10 n^{3} \sigma_{1}^{*}(n)+c(n)\right\} .
$$


Proof. From the definition of $\sigma_{1}^{*}(n)$ and by suitable change of variables, we deduce that

$$
\begin{aligned}
& \sum_{k=1}^{n-1} k^{2} \sigma_{1}^{*}(k) \sigma_{1}^{*}(n-k) \\
& =\sum_{k=1}^{n-1} k^{2}\left\{\sigma_{1}(k)-\sigma_{1}\left(\frac{k}{2}\right)\right\}\left\{\sigma_{1}(n-k)-\sigma_{1}\left(\frac{n-k}{2}\right)\right\} \\
& =\sum_{k=1}^{n-1} k^{2} \sigma_{1}(k) \sigma_{1}(n-k)-\sum_{k<\frac{n}{2}}(n-2 k)^{2} \sigma_{1}(n-2 k) \sigma_{1}(k) \\
& \quad+\sum_{k<\frac{n}{2}}(2 k)^{2} \sigma_{1}(k) \sigma_{1}(n-2 k)+\sum_{k<\frac{n}{2}}(2 k)^{2} \sigma_{1}(k) \sigma_{1}\left(\frac{n}{2}-k\right) .
\end{aligned}
$$

Thus the above equation can be written as

$$
\begin{aligned}
& =\sum_{k=1}^{n-1} k^{2} \sigma_{1}(k) \sigma_{1}(n-k)-n^{2} \sum_{k<\frac{n}{2}} \sigma_{1}(n-2 k) \sigma_{1}(k) \\
& \quad+4 n \sum_{k<\frac{n}{2}} k \sigma_{1}(n-2 k) \sigma_{1}(k)-8 \sum_{k<\frac{n}{2}} k^{2} \sigma_{1}(k) \sigma_{1}(n-2 k) \\
& \quad+4 \sum_{k<\frac{n}{2}} k^{2} \sigma_{1}(k) \sigma_{1}\left(\frac{n}{2}-k\right) .
\end{aligned}
$$

Therefore the Lemma follows from Lemma 4.11 and the result

$$
\sum_{k=1}^{n-1} k^{2} \sigma_{1}(k) \sigma_{1}(n-k)=\frac{1}{8} n^{2} \sigma_{3}(n)+\left(\frac{1}{24} n^{2}-\frac{1}{6} n^{3}\right) \sigma_{1}(n)
$$

of [31, p. 155].

Corollary 4.13. For any integer $M \geq 2$ with $M=p_{1}^{e_{1}} \cdots p_{r}^{e_{r}}$, we define $\operatorname{ord}_{p_{j}} M:=e_{j}$.

(i) Let $n$ be an odd integer. Then we have

$$
c(n) \equiv 6 n^{2} \sigma_{3}(n)-5 n^{3} \sigma_{1}(n) \quad(\bmod 960) .
$$

(ii) Let $n \in \mathbb{N}$. Then we have

$$
c(n) \equiv-9 n^{2} \sigma_{3}^{*}(n)+10 n^{3} \sigma_{1}^{*}(n) \quad(\bmod 120) .
$$

In particular, if $n$ is odd or $\operatorname{ord}_{p} n$ is odd for an odd prime $p$, then

$$
c(n) \equiv-9 n^{2} \sigma_{3}^{*}(n)+10 n^{3} \sigma_{1}^{*}(n) \quad(\bmod 240)
$$


and if $4 \mid n$ then

$$
c(n) \equiv-9 n^{2} \sigma_{3}^{*}(n)+10 n^{3} \sigma_{1}^{*}(n) \quad(\bmod 960) .
$$

Proof. (i) Since $c(1)=1$, we have

$$
c(1)=6 \cdot 1^{2} \sigma_{3}(1)-5 \cdot 1^{3} \sigma_{1}(1) \equiv 1 \quad(\bmod 960) .
$$

For an odd integer $n \geq 3$, it is obvious from (i) of Lemma 4.11.

(ii) By Lemma 4.12, we have

$$
120 \sum_{k=1}^{n-1} k^{2} \sigma_{1}^{*}(k) \sigma_{1}^{*}(n-k)=9 n^{2} \sigma_{3}^{*}(n)-10 n^{3} \sigma_{1}^{*}(n)+c(n) .
$$

Thus

$$
c(n) \equiv-9 n^{2} \sigma_{3}^{*}(n)+10 n^{3} \sigma_{1}^{*}(n) \quad(\bmod 120) .
$$

If $n$ is odd then the left hand side of (21) is

$$
\begin{aligned}
& 120\left\{\sum_{\substack{k=1 \\
2 \mid k}}^{n-1} k^{2} \sigma_{1}^{*}(k) \sigma_{1}^{*}(n-k)+\sum_{\substack{k=1 \\
2 \nmid k}}^{n-1} k^{2} \sigma_{1}^{*}(k) \sigma_{1}^{*}(n-k)\right\} \\
& =120\left\{\sum_{\substack{k=1 \\
2 \mid k}}^{n-1} k^{2} \sigma_{1}^{*}(k) \sigma_{1}^{*}(n-k)+\sum_{\substack{k=1 \\
2 \mid(n-k)}}^{n-1} k^{2} \sigma_{1}^{*}(k) \sigma_{1}^{*}(n-k)\right\} \\
& =240\left\{\sum_{\substack{k=1 \\
2 \mid k}}^{n-1} k^{2} \sigma_{1}^{*}\left(\frac{k}{2}\right) \sigma_{1}^{*}(n-k)+\sum_{\substack{k=1 \\
2 \mid(n-k)}}^{n-1} k^{2} \sigma_{1}^{*}(k) \sigma_{1}^{*}\left(\frac{n-k}{2}\right)\right\} .
\end{aligned}
$$

Note that $\sigma_{1}^{*}(k)=2 \sigma_{1}^{*}\left(\frac{k}{2}\right)$ for $2 \mid k$. Therefore, for odd $n$, we have

$$
c(n) \equiv-9 n^{2} \sigma_{3}^{*}(n)+10 n^{3} \sigma_{1}^{*}(n) \quad(\bmod 240) .
$$

Let $\operatorname{ord}_{p} n$ be odd. For odd $n$, we have already considered in (23). Thus let $n=2^{m} p^{s} p_{1}^{e_{1}} \cdots p_{r}^{e_{r}}$ with odd $s$ for an odd prime $p$ and $m \geq 1$. Then the left hand side of (21) is 
(24)

$$
\begin{aligned}
& 120 \sum_{k=1}^{n-1} k^{2} \sigma_{1}^{*}(k) \sigma_{1}^{*}(n-k) \\
& =120\left\{\sum_{k<\frac{n}{2}}\left(n^{2}+2 k^{2}-2 k n\right) \sigma_{1}^{*}(k) \sigma_{1}^{*}(n-k)+\left(\frac{n}{2}\right)^{2} \sigma_{1}^{*}\left(\frac{n}{2}\right) \sigma_{1}^{*}\left(\frac{n}{2}\right)\right\} \\
& =120\left[2 \sum_{k<\frac{n}{2}}\left(\frac{n^{2}}{2}+k^{2}-k n\right) \sigma_{1}^{*}(k) \sigma_{1}^{*}(n-k)+\left(\frac{n}{2}\right)^{2}\left\{\sigma_{1}^{*}\left(2^{m-1} p^{s} p_{1}^{e_{1}} \cdots p_{r}^{e_{r}}\right)\right\}^{2}\right] .
\end{aligned}
$$

Using $\sigma_{1}^{*}\left(2^{m-1} p^{s} p_{1}^{e_{1}} \cdots p_{r}^{e_{r}}\right)=\sigma_{1}^{*}\left(p^{s}\right) \sigma_{1}^{*}\left(2^{m-1} p_{1}^{e_{1}} \cdots p_{r}^{e_{r}}\right)$ and

$$
\begin{aligned}
\sigma_{1}^{*}\left(p^{s}\right) & =1+p+p^{2}+\cdots+p^{s} \\
& \equiv 0 \quad(\bmod 2),
\end{aligned}
$$

since $p$ odd and $s$ odd. Therefore, we can write $\sigma_{1}^{*}\left(\frac{n}{2}\right)=2 l$ for some $l \in \mathbb{N}$. So $(24)$ is

$$
240\left[\sum_{k<\frac{n}{2}}\left(\frac{n^{2}}{2}+k^{2}-k n\right) \sigma_{1}^{*}(k) \sigma_{1}^{*}(n-k)+2 l^{2}\left(\frac{n}{2}\right)^{2}\right] .
$$

Therefore

$$
c(n) \equiv-9 n^{2} \sigma_{3}^{*}(n)+10 n^{3} \sigma_{1}^{*}(n) \quad(\bmod 240) .
$$

Let us consider the case $4 \mid n$, i.e., $n=4 l$. Then the left hand side of $(21)$ is

(25)

$$
\begin{aligned}
& 120 \sum_{k=1}^{n-1} k^{2} \sigma_{1}^{*}(k) \sigma_{1}^{*}(n-k) \\
& =120\left\{\sum_{k<\frac{n}{2}} k^{2} \sigma_{1}^{*}(k) \sigma_{1}^{*}(n-k)+\sum_{\frac{n}{2}<k<n} k^{2} \sigma_{1}^{*}(k) \sigma_{1}^{*}(n-k)+\left(\frac{n}{2}\right)^{2} \sigma_{1}^{*}\left(\frac{n}{2}\right) \sigma_{1}^{*}\left(\frac{n}{2}\right)\right\} \\
& =120\left\{\sum_{k<\frac{n}{2}} k^{2} \sigma_{1}^{*}(k) \sigma_{1}^{*}(n-k)+\sum_{k<\frac{n}{2}}(n-k)^{2} \sigma_{1}^{*}(n-k) \sigma_{1}^{*}(k)+\left(\frac{n}{2}\right)^{2} \sigma_{1}^{*}\left(\frac{n}{2}\right) \sigma_{1}^{*}\left(\frac{n}{2}\right)\right\} \\
& =120\left\{\sum_{k<\frac{n}{2}}\left(n^{2}+2 k^{2}-2 k n\right) \sigma_{1}^{*}(k) \sigma_{1}^{*}(n-k)+n^{2} \sigma_{1}^{*}\left(\frac{n}{4}\right) \sigma_{1}^{*}\left(\frac{n}{4}\right)\right\} \\
& =240\left\{\sum_{k<2 l}\left(8 l^{2}+k^{2}-4 k l\right) \sigma_{1}^{*}(k) \sigma_{1}^{*}(4 l-k)+8 l^{2} \sigma_{1}^{*}(l) \sigma_{1}^{*}(l)\right\} .
\end{aligned}
$$


Thus if we can show $\sum_{k<2 l} k^{2} \sigma_{1}^{*}(k) \sigma_{1}^{*}(4 l-k) \equiv 0(\bmod 4)$ in $(25)$, then we can conclude that

$$
c(n) \equiv-9 n^{2} \sigma_{3}^{*}(n)+10 n^{3} \sigma_{1}^{*}(n) \quad(\bmod 960) .
$$

So we investigate the $\operatorname{sum} \sum_{k<2 l} k^{2} \sigma_{1}^{*}(k) \sigma_{1}^{*}(4 l-k)$. If $k \equiv 0$ $(\bmod 2)$, then $k^{2} \sigma_{1}^{*}(k) \sigma_{1}^{*}(4 l-k) \equiv 0(\bmod 4)$. Next suppose that $k \equiv 1(\bmod 2)$. If $k \equiv 1(\bmod 4)$ then $4 l-k \equiv 3(\bmod 4)$ and if $k \equiv 3(\bmod 4)$ then $4 l-k \equiv 1(\bmod 4)$. For integers $n \geq 3$ with $n \equiv 3(\bmod 4)$, we observe the fact that there exists a prime $p$ satisfying $p \mid n, \quad p \equiv 3(\bmod 4)$, and $\operatorname{ord}_{p} n=e_{i}$ with $e_{i}$ is odd. It is easily checked that

$$
\sigma_{1}^{*}\left(p^{e_{i}}\right) \equiv 1+p+p^{2}+\cdots+p^{e_{i}} \equiv 0 \quad(\bmod 4) .
$$

Since $\sigma_{1}^{*}\left(p^{e_{i}}\right) \mid \sigma_{1}^{*}(n)$, we have $\sigma_{1}^{*}(n) \equiv 0(\bmod 4)$. Applying this property, we infer that $\sigma_{1}^{*}(4 l-k) \equiv 0(\bmod 4)$ or $\sigma_{1}^{*}(k) \equiv 0$ $(\bmod 4)$. Thus we have $k^{2} \sigma_{1}^{*}(k) \sigma_{1}^{*}(4 l-k) \equiv 0(\bmod 4)$ for any positive integer $k$ satisfying $k<2 l$. Thus the claim

$$
c(n) \equiv-9 n^{2} \sigma_{3}^{*}(n)+10 n^{3} \sigma_{1}^{*}(n) \quad(\bmod 960)
$$

follows.

Theorem 4.14. Let $n \in \mathbb{N}$. Then we have

$$
\sum_{k=1}^{4 n-1} \sigma_{3}^{*}(k) \sigma_{3}^{*}(4 n-k) \equiv 8\left(n \sigma_{1}^{*}(n)-\sigma_{3}^{*}(n)\right) \quad(\bmod 24) .
$$

Proof. By Corollary 4.9, we obtain

$$
\begin{aligned}
& \sum_{k=1}^{4 n-1} \sigma_{3}^{*}(k) \sigma_{3}^{*}(4 n-k) \\
& \equiv \sum_{k=1}^{4 n-1}(3 k-2) \sigma_{1}^{*}(k)\left[\{3(4 n-k)-2\} \sigma_{1}^{*}(4 n-k)\right] \quad(\bmod 24) \\
& \equiv \sum_{k=1}^{4 n-1}\left(36 n k-9 k^{2}+4\right) \sigma_{1}^{*}(k) \sigma_{1}^{*}(4 n-k) \quad(\bmod 24) .
\end{aligned}
$$

Note that

$$
\sum_{k=1}^{4 n-1} k \sigma_{1}^{*}(k) \sigma_{1}^{*}(4 n-k)=\sum_{k=1}^{4 n-1}(4 n-k) \sigma_{1}^{*}(4 n-k) \sigma_{1}^{*}(k)
$$


and hence

$$
\sum_{k=1}^{4 n-1} k \sigma_{1}^{*}(k) \sigma_{1}^{*}(4 n-k)=2 n \sum_{k=1}^{4 n-1} \sigma_{1}^{*}(k) \sigma_{1}^{*}(4 n-k) .
$$

Thus we conclude that

$36 n \sum_{k=1}^{4 n-1} k \sigma_{1}^{*}(k) \sigma_{1}^{*}(4 n-k)=72 n^{2} \sum_{k=1}^{4 n-1} \sigma_{1}^{*}(k) \sigma_{1}^{*}(4 n-k) \equiv 0 \quad(\bmod 24)$.

Therefore,

$$
\begin{aligned}
& \sum_{k=1}^{4 n-1} \sigma_{3}^{*}(k) \sigma_{3}^{*}(4 n-k) \\
& \equiv-9 \sum_{k=1}^{4 n-1} k^{2} \sigma_{1}^{*}(k) \sigma_{1}^{*}(4 n-k)+4 \sum_{k=1}^{4 n-1} \sigma_{1}^{*}(k) \sigma_{1}^{*}(4 n-k) \quad(\bmod 24) \\
& \equiv-\frac{9}{120}\left\{9(4 n)^{2} \sigma_{3}^{*}(4 n)-10(4 n)^{3} \sigma_{1}^{*}(4 n)+c(4 n)\right\} \\
& \quad+4 \cdot \frac{1}{4}\left\{\sigma_{3}^{*}(4 n)-4 n \sigma_{1}^{*}(4 n)\right\} \quad(\bmod 24)
\end{aligned}
$$

using Lemma 4.12 and Table 5. By (ii) of Corollary 4.13, we find that if $4 \mid n$ then $c(n)=-9 n^{2} \sigma_{3}^{*}(n)+10 n^{3} \sigma_{1}^{*}(n)+960 L$ for some $L \in \mathbb{Z}$. Thus, we get

$$
\begin{aligned}
\sum_{k=1}^{4 n-1} \sigma_{3}^{*}(k) \sigma_{3}^{*}(4 n-k) & \equiv \sigma_{3}^{*}(4 n)-4 n \sigma_{1}^{*}(4 n) \quad(\bmod 24) \\
& \equiv 64 \sigma_{3}^{*}(n)-16 n \sigma_{1}^{*}(n) \quad(\bmod 24) .
\end{aligned}
$$

Therefore, the theorem follows.

From Theorem 4.14 and Corollary 4.9, it is easy to obtain :

Corollary 4.15. If $n$ is any prime other than 2 and 3 or any integer congruent to $1(\bmod 3)$, then we have

$$
\sum_{k=1}^{4 n-1} \sigma_{3}^{*}(k) \sigma_{3}^{*}(4 n-k) \equiv 0 \quad(\bmod 24) .
$$




\section{Appendix}

The table below gives values of $c_{8}(n)$ for $1 \leq n \leq 30$ and $n \in \mathbb{N}$.

\begin{tabular}{|c|c||c|c||c|c|}
\hline$n$ & $c_{8}(n)$ & $n$ & $c_{8}(n)$ & $n$ & $c_{8}(n)$ \\
\hline 1 & 1 & 11 & -44 & 21 & -96 \\
\hline 2 & 0 & 12 & 0 & 22 & 0 \\
\hline 3 & -4 & 13 & 22 & 23 & -56 \\
\hline 4 & 0 & 14 & 0 & 24 & 0 \\
\hline 5 & -2 & 15 & 8 & 25 & -121 \\
\hline 6 & 0 & 16 & 0 & 26 & 0 \\
\hline 7 & 24 & 17 & 50 & 27 & 152 \\
\hline 8 & 0 & 18 & 0 & 28 & 0 \\
\hline 9 & -11 & 19 & 44 & 29 & 198 \\
\hline 10 & 0 & 20 & 0 & 30 & 0 \\
\hline
\end{tabular}

TABLE 6 . Examples $c_{8}(n)(1 \leq n \leq 30)$

Acknowledgements: The author Ayyadurai Sankaranarayanan wishes to thank the National Institute for Mathematical Sciences (NIMS), Daejeon, Republic of Korea for its warm hospitality and generous support.

\section{References}

[1] A. Alaca, S. Alaca, E. McAfee, and K. S. Williams, Lambert series and Liouville's identities, Dissertationes Math. 445 (2007), 1-72.

[2] A. Alaca, S. Alaca and K. S. Williams, The convolution sums $\sum_{l+24 m=n} \sigma(l) \sigma(m)$ and $\sum_{3 l+8 m=n} \sigma(l) \sigma(m)$, M. J. Okayama Univ. 49 (2007), 93-111.

[3] A. Alaca, S. Alaca and K. S. Williams, The convolution sum $\sum_{m<n / 16} \sigma(m) \sigma(n-$ $16 m$ ), Canad. Math. Bull. 51 (2008), 3-14.

[4] B. C. Berndt, Ramanujan's Notebooks, Part II. Springer-Verlag, New York, 1989.

[5] N. Cheng and K. S. Williams, Evaluation of some convolution sums involving the sum of divisors functions, Yokohama Mathematical J., 52 (2005), 39-57.

[6] B. Cho, D. Kim and J.-K. Koo, Modular forms arising from divisor functions, J. Math. Anal. Appl. 356 (2009), 537-547.

[7] B. Cho, D. Kim and J.-K. Koo, Divisor functions arising from q-series, Publ. Math. Debrecen 76 (2010), 495-508.

[8] B. Cho, D. Kim, and H. Park, Evaluation of a certain combinatorial convolution sum in higher level cases, J. Math. Anal. Appl. 406 (2013), 203-210.

[9] L. E. Dickson, History of the Theory of Numbers, Vol.I, Chelsea Publ.Co., New York, 1952.

[10] J. W. L. Glaisher, On the square of the series in which the coefficients are the sums of the divisors of the exponents, Mess. Math. 14 (1884), 156-163.

[11] J. W. L. Glaisher, On certain sums of products of quantities depending upon the divisors of a number, Mess. Math. 15 (1885), 1-20. 
[12] J. W. L. Glaisher, Expressions for the five powers of the series in which the coefficients are the sums of the divisors of the exponents, Mess. Math. 15 (1885), $33-36$.

[13] H. Hahn, Convolution sums of some functions on divisors, Rocky Mountain J. Math. 37 (2007), 1593-1622.

[14] J. G. Huard, Z. M. Ou, B. K. Spearman, and K. S. Williams, Elementary Evaluation of Certain Convolution Sums Involving Divisor Functions, Number theory for the millennium, II, (2002), 229-274.

[15] C. G. J. Jacobi, Fundamenta Nova Theoriae Functionum Ellipticarum, Sumptibus Fratrum Bornträger ; reprinted in C. G. J. Jacobi, (1881-1891) Gesammelte Werke(Reimer, Berlin), Vol. 1, 49-239

[16] M. KANEKo AND D. ZaGieR, A generalized Jacobi theta function and quasimodular form, The moduli space of curves (Texel Island, 1994). Progr. Math. 129 Birkhäuser, 1995, 165-172.

[17] D. Kim, A. Kim, H. Park, Congruences of the Weierstrass $\wp(x)$ and $\wp^{\prime \prime}(x)(x=$ $\left.\frac{1}{2}, \frac{\tau}{2}, \frac{\tau+1}{2}\right)$ - functions on divisors, Bull. Korean Math. Soc. 50(1) (2013), 241261 .

[18] D. Kim, A. Kim, and N. Y. Ikikardes, Bernoulli numbers and certain convolution sums with divisor functions, Advances in Difference Equations 2013, 2013:277.

[19] D. Kim, M.-S. Kim, Divisor functions and Weierstrass functions arising from q-series, Bull. Korean Math. Soc., bf 49(4) (2012), 693-704.

[20] J. Levitt, On a Problem of Ramanujan, M. Phil thesis, University of Nottingham, 1978.

[21] J. Liouville, Sur quelques formules générales qui peuvent être utiles dans la théorie des nombres, J. Math. Pures App. 3 (1858), 241-250.

[22] J. Liouville, Sur quelques formules générales qui peuvent être utiles dans la theéorie des nombres, J. Math. Pures App. 3 (1858), 273-288

[23] E. McAfee, A three term arithmetic formula fo Lioville type with application to sums of six squares, M-sc thesis, Carleton University, Ottawa, Canada 2004.

[24] E. McAfee and K. S. Williams, An arithmetic formula of Liouville type and an extension of an identity of Ramanujan, JP J. Algebra Number Theory Appl, 6 (2006), 33-56.

[25] E. Meissner, Über die zahlentheoretischen Formuln Liouville's, Vierteljahrsschrift Naturforschende Gresellschaft in Zurich, 52 (1907), 156-216.

[26] G. Melfi, On some modular identities, de Gruyter, Berlin, 1998, 371-382.

[27] T. Pepin, Sur quelques formules d'analyse utiles dans la théorie des nombres, J. Math. Pures Appl. 4 (1888), 83-127.

[28] C. M. Piuma, Dimostrazione di alcune formole del Sig. Liouville, Giornale di Mat. 4 (1866), 1-14, 65-75, 193-201.

[29] S. Ramanujan, On certain arithmetical functions, Trans. Cambridge Philos. Soc. 22 (1916), 159-184.

[30] Whittaker, E. T. and G. N. Watson, A course of Modern Analysis, Cambridge Univ. Press 4th Ed., (1927), 464-498.

[31] K. S. Williams, Number Theory in the Spirit of Liouville, London Mathematical Society, Student Texts 76, Cambridge, 2011. 
Aeran Kim

Department of Mathematics and Institute of Pure and Applied Mathematics, Chonbuk National University,

Chonju, Chonbuk 561-756, Korea.

E-mail: ae_ran_kim@hotmail.com

Daeyeoul Kim

National Institute for Mathematical Sciences,

Yuseong-daero 1689-gil Yuseong-gu, Daejeon 305-811, South Korea.

E-mail: daeyeoul@nims.re.kr

Ayyadurai Sankaranarayanan

School of Mathematics, Tata Institute of Fundamental Research, Homi Bhabha Road, Mumbai 400005, India.

E-mail: sank@math.tifr.res.in 